\title{
PARAMETER-ROBUST STABILITY OF CLASSICAL THREE-FIELD FORMULATION OF BIOT'S CONSOLIDATION MODEL*
}

\author{
QINGGUO HONG ${ }^{\dagger}$ AND JOHANNES KRAUS $\ddagger$
}

\begin{abstract}
This paper is devoted to the stability analysis of a classical three-field formulation of Biot's consolidation model where the unknown variables are the displacements, fluid flux (Darcy velocity), and pore pressure. Specific parameter-dependent norms provide the key in establishing the full parameter-robust inf-sup stability of the continuous problem. Therefore, the stability results presented here are uniform not only with respect to the Lamé parameter $\lambda$, but also with respect to all the other model parameters. This allows for the construction of a uniform block diagonal preconditioner within the framework of operator preconditioning. Stable discretizations that meet the required conditions for full robustness and guarantee mass conservation strongly, i.e., pointwise, are discussed and corresponding optimal error estimates proved.
\end{abstract}

Key words. Biot's consolidation model, three-field formulation, parameter-robust stability, conservative discretizations, uniform preconditioners, optimal error estimates

AMS subject classifications. 65F10, 65N20, 65N30

1. Introduction: Biot's consolidation model. Poroelastic models describe mechanical deformation and fluid flow in porous media. They have a wide range of applications in medicine, biophysics, and geosciences such as the computation of intracranial pressure, trabecular bone stiffness under different loading conditions, reservoir simulation, waste repository performance, $\mathrm{CO}_{2}$ sequestration, consolidation of soil under surface loads, subsidence due to fluid withdrawal, and many others; see, e.g., [15, 35, 36, 37].

A classical and widely used model has been introduced by Biot [7, 8] and is based on the following assumptions:

(i) the porous medium is saturated by fluid and the temperature is constant,

(ii) the fluid in the porous medium is (nearly) incompressible,

(iii) the solid skeleton (matrix) is formed by an elastic material, and the deformations and strains are relatively small, and

(iv) the fluid flow is driven by Darcy's law (laminar flow).

For homogeneous isotropic linear elastic porous media, the Biot model in an open domain $\Omega \subset \mathbb{R}^{d}, d=2,3$, comprises the following system of partial differential equations (PDEs):

$$
\begin{aligned}
-\operatorname{div} \boldsymbol{\sigma}+c_{u p} \nabla p=\boldsymbol{f} & \text { in } \Omega \times(0, T), \\
\boldsymbol{v}=-\kappa \nabla p & \text { in } \Omega \times(0, T), \\
-c_{p u} \operatorname{div} \dot{\boldsymbol{u}}-\operatorname{div} \boldsymbol{v}-c_{p p} \dot{p}=g & \text { in } \Omega \times(0, T), \\
\boldsymbol{\epsilon}(\boldsymbol{u})=\frac{1}{2}\left(\nabla \boldsymbol{u}+(\nabla \boldsymbol{u})^{T}\right), & \\
\boldsymbol{\sigma}=2 \mu \boldsymbol{\epsilon}(\boldsymbol{u})+\lambda \operatorname{div}(\boldsymbol{u}) \boldsymbol{I} . &
\end{aligned}
$$

Here $\lambda$ and $\mu$ denote the Lamé parameters which are defined by

$$
\lambda:=\frac{\nu E}{(1+\nu)(1-2 \nu)}, \quad \mu:=\frac{E}{2(1+\nu)}
$$

* Received January 12, 2018. Accepted May 7, 2018. Published online on June 20, 2018. Recommended by Ulrich Langer.

${ }^{\dagger}$ Department of Mathematics, Pennsylvania State University, State College, PA, 16802, USA (huq11@psu. edu).

${ }^{\ddagger}$ Faculty of Mathematics, University of Duisburg-Essen, Thea-Leymann-Str. 9, 45127 Essen, Germany (johannes.kraus@uni-due.de). 
in terms of the modulus of elasticity (Young's modulus) $E$ and the Poisson ratio $\nu \in[0,1 / 2$ ).

The constant $c_{u p}=c_{p u}=\alpha$ that couples the pore pressure $p$ and the displacement variable $\boldsymbol{u}$ is the Biot-Willis constant, while $\kappa$ is the hydraulic conductivity given by the quotient between the permeability of the porous medium and the viscosity of the fluid. All constants are global constants here, i.e., parameters are assumed to be constant over the entire domain $\Omega$ in this paper. $I$ denotes the identity tensor, and $\sigma$ and $\epsilon$ are the effective stress and strain tensors, respectively, which are related to each other via the constitutive equation (1.1e). The strain tensor $\boldsymbol{\epsilon}(\boldsymbol{u})$ is given by the symmetric part of the gradient of the displacement field as defined in the compatibility condition (1.1d). The time derivatives of $\boldsymbol{u}$ and $p$ in the continuity equation (1.1c) are denoted by $\dot{\boldsymbol{u}}$ and $\dot{p}$. Finally, $\boldsymbol{v}$ denotes the fluid flux, sometimes also called percolation velocity of the fluid, which is assumed to be proportional to the (negative) pressure gradient as expressed by Darcy's law (1.1b). The right-hand side $f$ in the equilibrium equation (1.1a) represents the density of the applied body forces, and the source term $g$ in (1.1c) represents a forced fluid extraction or injection.

The system (1.1) is completed by proper boundary and initial conditions, e.g.,

$$
\begin{aligned}
p(\boldsymbol{x}, t) & =p_{D}(\boldsymbol{x}, t) & & \text { for } \boldsymbol{x} \in \Gamma_{p, D}, \quad t>0, \\
\boldsymbol{v}(\boldsymbol{x}, t) \cdot \boldsymbol{n}(\boldsymbol{x}) & =q_{N}(\boldsymbol{x}, t) & & \text { for } \boldsymbol{x} \in \Gamma_{p, N}, \quad t>0, \\
\boldsymbol{u}(\boldsymbol{x}, t) & =\boldsymbol{u}_{D}(\boldsymbol{x}, t) & & \text { for } \boldsymbol{x} \in \Gamma_{\boldsymbol{u}, D}, \quad t>0, \\
\left(\boldsymbol{\sigma}(\boldsymbol{x}, t)-c_{u p} p \boldsymbol{I}\right) \boldsymbol{n}(\boldsymbol{x}) & =\boldsymbol{g}_{N}(\boldsymbol{x}, t) & & \text { for } \boldsymbol{x} \in \Gamma_{\boldsymbol{u}, N}, \quad t>0,
\end{aligned}
$$

where $\Gamma_{p, D} \cap \Gamma_{p, N}=\emptyset, \bar{\Gamma}_{p, D} \cup \bar{\Gamma}_{p, N}=\Gamma=\partial \Omega$ and $\Gamma_{\boldsymbol{u}, D} \cap \Gamma_{\boldsymbol{u}, N}=\emptyset, \bar{\Gamma}_{\boldsymbol{u}, D} \cup \bar{\Gamma}_{\boldsymbol{u}, N}=\Gamma$. To complement the boundary conditions (1.2), the initial conditions at time $t=0$ have to satisfy (1.1a) and are given by

$$
\begin{array}{ll}
p(\boldsymbol{x}, 0)=p_{0}(\boldsymbol{x}) & \boldsymbol{x} \in \Omega, \\
\boldsymbol{u}(\boldsymbol{x}, 0)=\boldsymbol{u}_{0}(\boldsymbol{x}) & \boldsymbol{x} \in \Omega .
\end{array}
$$

Making use of the constitutive equation (1.1e) to eliminate the stress variable from the system results in the classical three-field formulation of the Biot model.

A common way to solve the time-dependent problem numerically is to discretize it in time and then solve a static problem in each time stamp. Using the backward Euler method for time discretization, one obtains a three-by-three block system of time-step equations

$$
\mathcal{A}\left[\begin{array}{c}
\boldsymbol{u}^{k} \\
\boldsymbol{v}^{k} \\
p^{k}
\end{array}\right]=\left[\begin{array}{c}
\boldsymbol{f}^{k} \\
\mathbf{0} \\
\tilde{g}^{k}
\end{array}\right]
$$

where

$$
\mathcal{A}:=\left[\begin{array}{ccc}
-2 \mu \operatorname{div} \epsilon-\lambda \nabla \operatorname{div} & 0 & c_{u p} \nabla \\
0 & \tau \kappa^{-1} I & \tau \nabla \\
-c_{p u} \operatorname{div} & -\tau \operatorname{div} & -c_{p p} I
\end{array}\right]
$$

for the unknown time-step functions

$$
\begin{aligned}
& \boldsymbol{u}^{k}=\boldsymbol{u}\left(\boldsymbol{x}, t_{k}\right) \in \boldsymbol{U}:=\left\{\boldsymbol{u} \in H^{1}(\Omega)^{d}: \boldsymbol{u}=\boldsymbol{u}_{D} \text { on } \Gamma_{\boldsymbol{u}, D}\right\} \\
& \boldsymbol{v}^{k}=\boldsymbol{v}\left(\boldsymbol{x}, t_{k}\right) \in \boldsymbol{V}:=\left\{\boldsymbol{v} \in H(\operatorname{div}, \Omega): \boldsymbol{v} \cdot \boldsymbol{n}=q_{N} \text { on } \Gamma_{p, N}\right\}, \\
& p^{k}=p\left(\boldsymbol{x}, t_{k}\right) \in P:=L^{2}(\Omega),
\end{aligned}
$$

and the right-hand side time-step functions

$$
\boldsymbol{f}^{k}=\boldsymbol{f}\left(\boldsymbol{x}, t_{k}\right) \quad \text { and } \quad \tilde{g}^{k}=\tau g\left(\boldsymbol{x}, t_{k}\right)-c_{p u} \operatorname{div}\left(\boldsymbol{u}^{k-1}\right)-c_{p p} p^{k-1}
$$


at any given time $t=t_{k}=t_{k-1}+\tau$. As in the remainder of this paper we will consider the static problem (1.3)-(1.4); for convenience, we will drop the superscript for the time-step functions: we replace $\boldsymbol{u}^{k}, \boldsymbol{v}^{k}$, and $p^{k}$ with $\boldsymbol{u}, \boldsymbol{v}$, and $p$, respectively.

Following the standard notation, $L^{2}(\Omega)$ denotes the space of square Lebesgue integrable functions equipped with the standard $L^{2}$-norm $\|\cdot\|$ and $H^{1}(\Omega)^{d}$ the space of vector-valued $H^{1}$-functions equipped with the norm $\|\cdot\|_{1}$ defined by $\|\boldsymbol{u}\|_{1}^{2}:=\|\boldsymbol{u}\|^{2}+\|\nabla \boldsymbol{u}\|^{2}$. Moreover, we define $H(\operatorname{div} ; \Omega):=\left\{\boldsymbol{v} \in L^{2}(\Omega)^{d}: \operatorname{div} \boldsymbol{v} \in L^{2}(\Omega)\right\}$ where the standard Sobolev norm $\|\cdot\|_{\text {div }}$ is $\|\boldsymbol{v}\|_{\text {div }}^{2}:=\|\boldsymbol{v}\|^{2}+\|\operatorname{div} \boldsymbol{v}\|^{2}$.

We will often consider the case that $\Gamma_{\boldsymbol{u}, D}=\Gamma_{p, N}=\Gamma$ and $\boldsymbol{u}_{D}=\mathbf{0}, q_{N}=0$, and we will thus write $\boldsymbol{U}=H_{0}^{1}(\Omega)^{d}$ and $\boldsymbol{V}=H_{0}(\operatorname{div}, \Omega)$. In this setting, in order to determine the solution for the pressure variable $p$ uniquely, one can set

$$
P=L_{0}^{2}(\Omega):=\left\{p \in L^{2}(\Omega): \int_{\Omega} p d \boldsymbol{x}=0\right\} .
$$

In many applications the variations of the model parameters are quite large. In geophysical applications, for example, the permeability typically varies in the range from $10^{-9}$ to $10^{-21} \mathrm{~m}^{2}$, whereas Young's modulus is typically in the order of GPa and the Poisson ratio in the range $0.1-0.3$; see $[14,27,37]$. The soft tissue of the central nervous system, on the other hand, has a permeability of about $10^{-14}$ to $10^{-16} \mathrm{~m}^{2}$, whereas Young's modulus is typically in the order of $\mathrm{kPa}$ and the Poisson ratio in the range 0.3 to almost 0.5 ; see $[35,36]$. For this reason it is important that both the formulation of the problem and the numerical methods for its solution are stable over the whole range of values of the parameters in the model.

The stability of the time discretization and space discretization by finite difference or finite volume methods have been studied in $[4,18,19,30]$ and will not be addressed here. Instead, we will focus on the issue of inf-sup stable finite element discretizations of the static problem (1.3)-(1.4). It is a well known fact that the LBB condition (see [5, 11]) plays a crucial role in the well-posedness analysis of the continuous problem and its discrete counterparts arising from mixed finite element discretizations. It is also the key tool in deriving a priori error estimates. The inf-sup stability for the Darcy problem, as well as for the Stokes and linear elasticity problems, is well understood, and various stable mixed discretizations of either of these systems of PDEs have been proposed over the years; see, e.g., [9] and references therein.

Biot's model of poroelasticity combines these equations, and, as we will see in the next sections, the parameter-robust stability of its three-field formulation becomes more delicate. Alternative formulations that can be proven to be stable independently of the model parameters (in certain norms) include a two-field formulation for the displacements and pore pressure (see [1]) and a new three-field formulation that—-besides the displacements—introduces two pressure unknowns, one for the fluid pressure and one for the total pressure, defined as a weighted sum of fluid and solid pressure [27].

Another formulation has recently been proposed and analyzed in [6]. The authors there use mixed methods based on the Hellinger-Reissner variational principle for the elasticity part of the system and impose weakly the symmetry of the stress tensor resulting in a saddle point problem for $\boldsymbol{\sigma}, \boldsymbol{u}, p$ and a Lagrange multiplier, for which they prove the parameter-robust stability of the resulting four-field formulation.

Compared to the new three-field formulation presented in [27, 31], the classic three-field formulation of Biot's consolidation model retains Darcy's law in order to guarantee fluid mass conservation. A four-field formulation in which the stress tensor is kept as an additional variable in the system has been proposed in [26], and the error analysis there is robust with respect to $\lambda$ but not uniform with respect to other parameters such as $\tau$ and $\kappa$.

Error estimates were obtained in $[32,33]$ coupling continuous or discontinuous Galerkin approximations of the solid displacement with a mixed method for the pressure but under 
the restrictive assumption $c_{p p}>0$. Nonconforming finite elements have been shown to be beneficial with regard to reducing pressure oscillations in computations based on the classical three-field formulation; see [24]. The lowest approximation order, consisting of Crouzeix-Raviart finite elements for the displacements, lowest-order Raviart-Thomas elements for the Darcy velocity, and a piecewise constant approximation of the pressure unknown in combination with a mass-lumping technique for the Raviart-Thomas elements results in a computationally efficient method. However, the norms defined in the latter work and in many others do not allow to establish the full parameter-robust stability we aim for.

In the present paper, we establish full parameter-robust stability for the classic threefield formulation of Biot's consolidation model. Crucial in our analysis is the definition of proper norms for which we prove that the constants in the related boundedness and inf-sup conditions do not depend on any of the model parameters. We also want to emphasize that our stability results cover both the case of an incompressible Newtonian fluid $\left(c_{p p}=0\right)$ and the case of the constrained specific storage coefficient $c_{p p}$ being positive. Further, we propose a discretization that fulfills mass conservation strongly at a discrete level. We also prove the full parameter-robust stability of the discretized problem and the related optimal error estimates. The remainder of the paper is organized as follows.

In Section 2, we briefly revisit non-uniform stability results and make some useful observations which motivate the subsequent analysis. In Section 3 we introduce the parameterdependent norms based on which we establish the parameter-robust stability of the weak formulation of the continuous problem (1.3)-(1.4). In Section 4 we analyze mixed finite element discretizations that provide discrete parameter-robust inf-sup stability and strong mass conservation. Applying the theory of operator preconditioning, see [29], the results from Sections 3 and 4 imply the uniformity (parameter-robustness) of the (canonical) normequivalent block-diagonal preconditioners. In Section 5 we use our findings to derive robust optimal a priori error estimates. Finally, Section 6 gives some concluding remarks.

Throughout this paper, the hidden constants in $\lesssim, \gtrsim$ and $\approx$ are independent of the parameters $\mu, \lambda, c_{u p}, \tau, \kappa, c_{p u}, c_{p p}$ and the mesh size $h$.

2. A revisit of non-uniform stability results. We begin our stability analysis recasting equations (1.3)-(1.4). We first eliminate the parameter $\mu$ from the model (1.3)-(1.4) by dividing all the parameters by $2 \mu$, that is, we make the substitutions

$$
2 \mu \rightarrow 1, \lambda / 2 \mu \rightarrow \lambda, c_{u p} / 2 \mu \rightarrow c_{u p}, \boldsymbol{f} / 2 \mu \rightarrow \boldsymbol{f}, \tau / 2 \mu \rightarrow \tau, c_{p p} / 2 \mu \rightarrow c_{p p}, g / 2 \mu \rightarrow g .
$$

Herewith, the system (1.3) becomes

$$
\begin{aligned}
-\operatorname{div} \boldsymbol{\epsilon}(\boldsymbol{u})-\lambda \nabla \operatorname{div} \boldsymbol{u}+c_{u p} \nabla p & =\boldsymbol{f}, \\
\tau \kappa^{-1} \boldsymbol{v}+\tau \nabla p & =\mathbf{0}, \\
-c_{p u} \operatorname{div} \boldsymbol{u}-\tau \operatorname{div} \boldsymbol{v}-c_{p p} p & =g .
\end{aligned}
$$

Now let $\tilde{\boldsymbol{u}}=c_{p u} \boldsymbol{u}, \tilde{\boldsymbol{v}}=\tau \boldsymbol{v}, \tilde{p}=c_{p u}^{2} p, \tilde{\boldsymbol{f}}=c_{p u} \boldsymbol{f}$ and divide the second equation in (2.1) by $\tau$ to get

$$
\begin{aligned}
-\operatorname{div} \boldsymbol{\epsilon}(\tilde{\boldsymbol{u}})-\lambda \nabla \operatorname{div} \tilde{\boldsymbol{u}}+\nabla \tilde{p} & =\tilde{\boldsymbol{f}}, \\
\alpha^{2} \tau^{-1} \kappa^{-1} \tilde{\boldsymbol{v}}+\nabla \tilde{p} & =\mathbf{0}, \\
-\operatorname{div} \tilde{\boldsymbol{u}}-\operatorname{div} \tilde{\boldsymbol{v}}-c_{p p} \alpha^{-2} \tilde{p} & =g,
\end{aligned}
$$

where we have also used that $c_{u p}=c_{p u}=\alpha$. For convenience we denote

$$
R_{p}^{-1}=\alpha^{2} \tau^{-1} \kappa^{-1}, \quad \alpha_{p}=c_{p p} \alpha^{-2}
$$


and further assume that the ranges of the parameters are

$$
\lambda \geq 1, \quad R_{p}^{-1}>0, \quad \alpha_{p} \geq 0 .
$$

These assumptions are very general and reasonable. In particular, they cover the case when the Lamé parameter $\lambda$ tends to infinity, the case when the permeability of the porous medium and/or the time step are arbitrarily small, the case of incompressible Newtonian fluids $\left(c_{p p}=0\right)$ as well as the case when the constrained specific storage coefficient $c_{p p}$ is positive.

In the following, in order to simplify the notation, we omit the "tilde" symbol, i.e., we write $\tilde{\boldsymbol{u}} \rightarrow \boldsymbol{u}, \tilde{\boldsymbol{v}} \rightarrow \boldsymbol{v}, \tilde{\boldsymbol{f}} \rightarrow \boldsymbol{f}, \tilde{p} \rightarrow p$. Therefore, without loss of generality, we can write the system in the form

$$
\begin{aligned}
-\operatorname{div} \boldsymbol{\epsilon}(\boldsymbol{u})-\lambda \nabla \operatorname{div} \boldsymbol{u}+\nabla p & =\boldsymbol{f} \\
R_{p}^{-1} \boldsymbol{v}+\nabla p & =\mathbf{0} \\
-\operatorname{div} \boldsymbol{u}-\operatorname{div} \boldsymbol{v}-\alpha_{p} p & =g
\end{aligned}
$$

or, in short notation,

$$
\mathcal{A}\left[\begin{array}{l}
\boldsymbol{u} \\
\boldsymbol{v} \\
p
\end{array}\right]=\left[\begin{array}{l}
\boldsymbol{f} \\
\mathbf{0} \\
g
\end{array}\right]
$$

where

$$
\mathcal{A}:=\left[\begin{array}{ccc}
-\operatorname{div} \epsilon-\lambda \nabla \operatorname{div} & 0 & \nabla \\
0 & R_{p}^{-1} I & \nabla \\
-\operatorname{div} & -\operatorname{div} & -\alpha_{p} I
\end{array}\right] .
$$

REMARK 2.1. In general, we assume that $\int_{\Omega} g d x=0$. When $\alpha_{p}=0$ this assumption is sometimes referred to as consistency condition. If $\alpha_{p}>0$ and $\int_{\Omega} g d x \neq 0$, then we can decompose $g$ as $g=g_{0}+g_{c}$, where $g_{0}=g-\frac{1}{|\Omega|} \int_{\Omega} g d x$ and $g_{c}=\frac{1}{|\Omega|} \int_{\Omega} g d x$, so that $\int_{\Omega} g_{0} d x=0$. It follows that the solution $(\boldsymbol{u}, \boldsymbol{v}, p)$ can be decomposed as $(\boldsymbol{u}, \boldsymbol{v}, p)=\left(\boldsymbol{u}, \boldsymbol{v}, p_{0}\right)+\left(\mathbf{0}, \mathbf{0}, p_{c}\right)$, where $p_{0} \in L_{0}^{2}(\Omega)$ and $p_{c}=\alpha_{p}^{-1} \frac{1}{|\Omega|} \int_{\Omega} g d x$. Hence, we still need to consider only the case when $\int_{\Omega} g d x=0$.

The weak formulation of (2.2) requires to find $(\boldsymbol{u}, \boldsymbol{v}, p) \in \boldsymbol{U} \times \boldsymbol{V} \times P$ such that, for any $(\boldsymbol{w}, \boldsymbol{z}, q) \in \boldsymbol{U} \times \boldsymbol{V} \times P$,

$$
\begin{aligned}
(\boldsymbol{\epsilon}(\boldsymbol{u}), \boldsymbol{\epsilon}(\boldsymbol{w}))+\lambda(\operatorname{div} \boldsymbol{u}, \operatorname{div} \boldsymbol{w})-(p, \operatorname{div} \boldsymbol{w}) & =(\boldsymbol{f}, \boldsymbol{w}), \\
R_{p}^{-1}(\boldsymbol{v}, \boldsymbol{z})-(p, \operatorname{div} \boldsymbol{z}) & =0, \\
-(\operatorname{div} \boldsymbol{u}, q)-(\operatorname{div} \boldsymbol{v}, q)-\alpha_{p}(p, q) & =(g, q) .
\end{aligned}
$$

Motivated by the work [28], let us first consider the Hilbert spaces $\boldsymbol{U}=H_{0}^{1}(\Omega)^{d}$, $\boldsymbol{V}=H_{0}(\operatorname{div}, \Omega)$, and $P=L_{0}^{2}(\Omega)$ with the (weighted) norms defined by

$$
\begin{aligned}
(\boldsymbol{u}, \boldsymbol{w})_{\boldsymbol{U}} & =(\boldsymbol{\epsilon}(\boldsymbol{u}), \boldsymbol{\epsilon}(\boldsymbol{w}))+\lambda(\operatorname{div} \boldsymbol{u}, \operatorname{div} \boldsymbol{w}), \\
(\boldsymbol{v}, \boldsymbol{z})_{\boldsymbol{V}} & =R_{p}^{-1}(\boldsymbol{v}, \boldsymbol{z})+R_{p}^{-1}(\operatorname{div} \boldsymbol{v}, \operatorname{div} \boldsymbol{z}), \\
(p, q)_{P} & =(p, q) .
\end{aligned}
$$

Before we study Biot's equations, we recall the following well known results; see, e.g., [9, 11]. 
LEMMA 2.2. There exists a positive constant $\beta_{d}>0$ such that

$$
\inf _{q \in P} \sup _{\boldsymbol{v} \in \boldsymbol{V}} \frac{(\operatorname{div} \boldsymbol{v}, q)}{\|\boldsymbol{v}\|_{\operatorname{div}}\|q\|} \geq \beta_{d} .
$$

LEMMA 2.3. There exists a positive constant $\beta_{s}>0$ such that

$$
\inf _{q \in P} \sup _{\boldsymbol{u} \in \boldsymbol{U}} \frac{(\operatorname{div} \boldsymbol{u}, q)}{\|\boldsymbol{u}\|_{1}\|q\|} \geq \beta_{s} .
$$

Let us now investigate the stability of the formulation (2.4). The conditions for the well-posedness of saddle-point problems of this type are well known; see, e.g., [9, 16]. In the following we want to comment on some issues related to the robustness of the inf-sup condition in the Banach-Necas-Babuska theorem (cf. [16]) under the norms induced by (2.5).

To illustrate these, let us consider two different cases, namely,

- Case (i): $\lambda \gg 1, R_{p}^{-1} \approx 1$, and $0 \leq \alpha_{p} \lesssim 1$ and

- Case (ii): $\lambda \approx 1, \alpha \approx 1, c_{p p} \bar{\sim} \alpha / \lambda$, and $\kappa$ or $\tau$ tending to zero.

In Case (i), for some nearly incompressible materials, we have that the Lamé parameter $\lambda$ tends to infinity. If we assume that $\lambda \gg 1, R_{p}^{-1} \approx 1$ and $0 \leq \alpha_{p} \lesssim 1$ then, defining the norms according to (2.5), the boundedness of both $(\boldsymbol{\epsilon}(\boldsymbol{u}), \boldsymbol{\epsilon}(\boldsymbol{w}))+\lambda(\operatorname{div} \boldsymbol{u}, \operatorname{div} \boldsymbol{w})+R_{p}^{-1}(\boldsymbol{v}, \boldsymbol{z})$ and $(\operatorname{div} \boldsymbol{u}, q)+(\operatorname{div} \boldsymbol{v}, q)$ is obvious. Further, for $0 \leq \alpha_{p} \lesssim 1$, we obtain the boundedness of $\alpha_{p}(p, q)$. Moreover, defining the norms by (2.5), using Lemma 2.2, and choosing $(\boldsymbol{u}, \boldsymbol{v})=(\mathbf{0}, \boldsymbol{v}) \in \boldsymbol{U} \times \boldsymbol{V}$, we obtain the inf-sup condition

$$
\inf _{q \in P} \sup _{(\boldsymbol{u}, \boldsymbol{v}) \in \boldsymbol{U} \times \boldsymbol{V}} \frac{(\operatorname{div} \boldsymbol{u}, q)+(\operatorname{div} \boldsymbol{v}, q)}{\left(\|\boldsymbol{u}\|_{\boldsymbol{U}}+\|\boldsymbol{v}\|_{\boldsymbol{V}}\right)\|q\|_{P}} \geq \beta_{d v}>0 .
$$

Finally, the coercivity of $(\boldsymbol{\epsilon}(\boldsymbol{u}), \boldsymbol{\epsilon}(\boldsymbol{u}))+\lambda(\operatorname{div} \boldsymbol{u}, \operatorname{div} \boldsymbol{u})+R_{p}^{-1}(\boldsymbol{v}, \boldsymbol{v})$ on the kernel set

$$
\boldsymbol{Z}=\{(\boldsymbol{u}, \boldsymbol{v}) \in \boldsymbol{U} \times \boldsymbol{V}:(\operatorname{div} \boldsymbol{u}, q)+(\operatorname{div} \boldsymbol{v}, q)=0, \forall q \in P\}
$$

can also be verified. Indeed, since $(\boldsymbol{u}, \boldsymbol{v}) \in \boldsymbol{Z}$ means $\operatorname{div} \boldsymbol{v}=-\operatorname{div} \boldsymbol{u}$, it follows that

$$
\begin{aligned}
(\boldsymbol{\epsilon}(\boldsymbol{u}), \boldsymbol{\epsilon}(\boldsymbol{u})) & +\lambda(\operatorname{div} \boldsymbol{u}, \operatorname{div} \boldsymbol{u})+R_{p}^{-1}(\boldsymbol{v}, \boldsymbol{v}) \\
& \geq(\boldsymbol{\epsilon}(\boldsymbol{u}), \boldsymbol{\epsilon}(\boldsymbol{u}))+\frac{\lambda}{2}(\operatorname{div} \boldsymbol{u}, \operatorname{div} \boldsymbol{u})+R_{p}^{-1}(\boldsymbol{v}, \boldsymbol{v})+\frac{\lambda}{2}(\operatorname{div} \boldsymbol{u}, \operatorname{div} \boldsymbol{u}) \\
& =(\boldsymbol{\epsilon}(\boldsymbol{u}), \boldsymbol{\epsilon}(\boldsymbol{u}))+\frac{\lambda}{2}(\operatorname{div} \boldsymbol{u}, \operatorname{div} \boldsymbol{u})+R_{p}^{-1}(\boldsymbol{v}, \boldsymbol{v})+\frac{\lambda}{2}(\operatorname{div} \boldsymbol{v}, \operatorname{div} \boldsymbol{v}) \\
& \gtrsim \frac{1}{2}\left((\boldsymbol{\epsilon}(\boldsymbol{u}), \boldsymbol{\epsilon}(\boldsymbol{u}))+\lambda(\operatorname{div} \boldsymbol{u}, \operatorname{div} \boldsymbol{u})+R_{p}^{-1}(\boldsymbol{v}, \boldsymbol{v})+R_{p}^{-1}(\operatorname{div} \boldsymbol{v}, \operatorname{div} \boldsymbol{v})\right)
\end{aligned}
$$

where the last inequality comes from the assumption $\lambda \gg 1, R_{p}^{-1} \approx 1$.

On the other hand, in many practical applications one is confronted with Case (ii). Then, since in this case we have $R_{p}^{-1} \gg 1$ and $0 \leq \alpha_{p} \lesssim 1$, defining the norms according to (2.5), the boundedness of both $(\boldsymbol{\epsilon}(\boldsymbol{u}), \boldsymbol{\epsilon}(\boldsymbol{w}))+\lambda(\operatorname{div} \boldsymbol{u}, \operatorname{div} \boldsymbol{w})+R_{p}^{-1}(\boldsymbol{v}, \boldsymbol{z})$ and $(\operatorname{div} \boldsymbol{u}, q)+(\operatorname{div} \boldsymbol{v}, q)$ are obvious again. Further, the assumption $0 \leq \alpha_{p} \lesssim 1$ implies the boundedness of $\alpha_{p}(p, q)$. Next, using Lemma 2.3 and choosing $(\boldsymbol{u}, \boldsymbol{v})=(\boldsymbol{u}, \mathbf{0}) \in \boldsymbol{U} \times \boldsymbol{V}$, we obtain the inf-sup condition

$$
\inf _{q \in P} \sup _{(\boldsymbol{u}, \boldsymbol{v}) \in \boldsymbol{U} \times \boldsymbol{V}} \frac{(\operatorname{div} \boldsymbol{u}, q)+(\operatorname{div} \boldsymbol{v}, q)}{\left(\|\boldsymbol{u}\|_{\boldsymbol{U}}+\|\boldsymbol{v}\|_{\boldsymbol{V}}\right)\|q\|_{P}} \geq \beta_{s u}>0
$$


under the norms induced by (2.5) since $\lambda \approx 1$. We see, however, that in this case the coercivity of $(\boldsymbol{\epsilon}(\boldsymbol{u}), \boldsymbol{\epsilon}(\boldsymbol{u}))+\lambda(\operatorname{div} \boldsymbol{u}, \operatorname{div} \boldsymbol{u})+R_{p}^{-1}(\boldsymbol{v}, \boldsymbol{v})$ on the kernel set $\boldsymbol{Z}$ defined at (2.6) cannot be valid any more. Indeed, since $(\boldsymbol{u}, \boldsymbol{v}) \in \boldsymbol{Z}$ means $\operatorname{div} \boldsymbol{v}=-\operatorname{div} \boldsymbol{u}$, it follows that for any $M>0$, there exits $(\boldsymbol{u}, \boldsymbol{v})$, where, e.g., $\operatorname{div} \boldsymbol{v} \neq 0$ and $R_{p}^{-1}$ is large enough such that

$$
\begin{aligned}
(\boldsymbol{\epsilon}(\boldsymbol{u}), \boldsymbol{\epsilon}(\boldsymbol{u}))+ & (\operatorname{div} \boldsymbol{u}, \operatorname{div} \boldsymbol{u})+R_{p}^{-1}(\boldsymbol{v}, \boldsymbol{v})+R_{p}^{-1}(\operatorname{div} \boldsymbol{v}, \operatorname{div} \boldsymbol{v}) \\
& \geq M\left((\boldsymbol{\epsilon}(\boldsymbol{u}), \boldsymbol{\epsilon}(\boldsymbol{u}))+(\operatorname{div} \boldsymbol{u}, \operatorname{div} \boldsymbol{u})+R_{p}^{-1}(\boldsymbol{v}, \boldsymbol{v})\right) \\
& \gtrsim M\left((\boldsymbol{\epsilon}(\boldsymbol{u}), \boldsymbol{\epsilon}(\boldsymbol{u}))+\lambda(\operatorname{div} \boldsymbol{u}, \operatorname{div} \boldsymbol{u})+R_{p}^{-1}(\boldsymbol{v}, \boldsymbol{v})\right),
\end{aligned}
$$

where the second inequality comes from $\lambda \lesssim 1$. Hence, in this case the estimate (2.7) implies that for any $M>0$, there exists $(\boldsymbol{u}, \boldsymbol{v}) \in \tilde{\boldsymbol{Z}}$ (and $R_{p}^{-1}$ large enough) such that

$$
\|\boldsymbol{u}\|_{\boldsymbol{U}}^{2}+\|\boldsymbol{v}\|_{\boldsymbol{V}}^{2} \geq M\left((\boldsymbol{\epsilon}(\boldsymbol{u}), \boldsymbol{\epsilon}(\boldsymbol{u}))+(\operatorname{div} \boldsymbol{u}, \operatorname{div} \boldsymbol{u})+R_{p}^{-1}(\boldsymbol{v}, \boldsymbol{v})\right) .
$$

Therefore, the system (2.4) is not uniformly stable with respect to the parameter $R_{p}^{-1}$ under the norms induced by (2.5).

From this observation we conclude that we have to define proper norms (as we do below in (3.2)) in order to establish the coercivity of $(\boldsymbol{\epsilon}(\boldsymbol{u}), \boldsymbol{\epsilon}(\boldsymbol{u}))+\lambda(\operatorname{div} \boldsymbol{u}, \operatorname{div} \boldsymbol{u})+R_{p}^{-1}(\boldsymbol{v}, \boldsymbol{v})$ on $Z$ in both the above cases.

3. Parameter-robust stability of the model. In this section, we first define proper parameter-dependent norms for the spaces $\boldsymbol{U}, \boldsymbol{V}$, and $P$ based on which we will then establish the parameter-robust stability of the Biot problem (2.4) for parameters in the ranges

$$
\lambda \geq 1, \quad R_{p}^{-1}>0, \quad \alpha_{p} \geq 0 .
$$

The ultimate goal and subject of ongoing and future research is parameter-robust stability of the problem (2.4) for nonsmooth coefficients, e.g., for piecewise (layerwise or even only elementwise) constant coefficients. In this work, however, we consider only the case of globally constant coefficients. We want to remark in this context that even for the Darcy problem, which is in some sense part of the considered three-field formulation of Biot's consolidation model, robust stability for the most general case of heterogeneous coefficients is an issue that is not fully resolved yet; see the discussion in [25] and the references therein.

Let us denote

$$
\rho=\min \left\{\lambda, R_{p}^{-1}\right\} \quad \text { and } \quad \gamma=\max \left\{\rho^{-1}, \alpha_{p}\right\},
$$

and let us consider the Hilbert spaces $\boldsymbol{U}=H_{0}^{1}(\Omega)^{d}, \boldsymbol{V}=H_{0}(\operatorname{div}, \Omega), P=L_{0}^{2}(\Omega)$ with parameter-dependent norms $\|\cdot\|_{U},\|\cdot\|_{V},\|\cdot\|_{P}$ induced by the inner products

$$
\begin{aligned}
(\boldsymbol{u}, \boldsymbol{w})_{\boldsymbol{U}} & =(\boldsymbol{\epsilon}(\boldsymbol{u}), \boldsymbol{\epsilon}(\boldsymbol{w}))+\lambda(\operatorname{div} \boldsymbol{u}, \operatorname{div} \boldsymbol{w}) \\
(\boldsymbol{v}, \boldsymbol{z})_{\boldsymbol{V}} & =R_{p}^{-1}(\boldsymbol{v}, \boldsymbol{z})+\gamma^{-1}(\operatorname{div} \boldsymbol{v}, \operatorname{div} \boldsymbol{z}) \\
(p, q)_{P} & =\gamma(p, q)
\end{aligned}
$$

As we will show in this section, the above norms are the key for establishing the parameterrobust stability of the model. 
REMARK 3.1. The Stokes-type inf-sup condition, namely Lemma 2.3, still holds and the norms can still be defined by (3.2) when both the measures of $\Gamma_{\boldsymbol{u}, D}$ and $\Gamma_{\boldsymbol{u}, N}$ are nonzero and the Hilbert spaces are given by $\boldsymbol{U}=H_{D}^{1}(\Omega)^{d}=\left\{\boldsymbol{u}: \boldsymbol{u} \in H^{1}(\Omega)^{d},\left.\boldsymbol{u}\right|_{\Gamma_{\boldsymbol{u}, D}}=\mathbf{0}\right\}$, $\boldsymbol{V}=H(\operatorname{div}, \Omega)$, and $P=L^{2}(\Omega)$. However, if $\Gamma_{\boldsymbol{u}, D}=\Gamma$ and the Hilbert spaces are given by $\boldsymbol{U}=H_{0}^{1}(\Omega)^{d}, \boldsymbol{V}=H(\operatorname{div}, \Omega)$, and $P=L^{2}(\Omega)$, then we have to modify the norms. In this case, we need to set $\rho=\min \left\{R_{p}^{-1}, \lambda\right\}, \gamma_{m}=\max \left\{\rho^{-1}, \alpha_{p}\right\}, \gamma_{0}=\max \left\{R_{p}, \alpha_{p}\right\}$ and define the norms by

$$
\begin{aligned}
(\boldsymbol{u}, \boldsymbol{w})_{\boldsymbol{U}} & =(\epsilon(\boldsymbol{u}), \epsilon(\boldsymbol{w}))+\lambda(\operatorname{div} \boldsymbol{u}, \operatorname{div} \boldsymbol{w}), \\
(\boldsymbol{v}, \boldsymbol{z})_{\boldsymbol{V}} & =R_{p}^{-1}(\boldsymbol{v}, \boldsymbol{z})+\gamma_{m}^{-1}\left(Q_{m} \operatorname{div} \boldsymbol{v}, Q_{m} \operatorname{div} \boldsymbol{z}\right)+\gamma_{0}^{-1}\left(Q_{0} \operatorname{div} \boldsymbol{v}, Q_{0} \operatorname{div} \boldsymbol{z}\right), \\
(p, q)_{P} & =\gamma_{m}\left(Q_{m} p, Q_{m} q\right)+\gamma_{0}\left(Q_{0} p, Q_{0} q\right),
\end{aligned}
$$

where $Q_{m}: L^{2}(\Omega) \rightarrow L_{0}^{2}(\Omega)$ denotes the $L^{2}$-projection, and $Q_{0}$ is the $L^{2}$-projection from $L^{2}(\Omega)$ to the space of globally constant functions. The proof of parameter robust stability then becomes more technical, and we will not present it here.

We introduce the bilinear form

$$
\begin{aligned}
& A((\boldsymbol{u}, \boldsymbol{v}, p),(\boldsymbol{w}, \boldsymbol{z}, q)) \\
& \quad(\boldsymbol{\epsilon}(\boldsymbol{u}), \boldsymbol{\epsilon}(\boldsymbol{w}))+\lambda(\operatorname{div} \boldsymbol{u}, \operatorname{div} \boldsymbol{w})-(p, \operatorname{div} \boldsymbol{w}) \\
& \quad+R_{p}^{-1}(\boldsymbol{v}, \boldsymbol{z})-(p, \operatorname{div} \boldsymbol{z})-(\operatorname{div} \boldsymbol{u}, q)-(\operatorname{div} \boldsymbol{v}, q)-\alpha_{p}(p, q),
\end{aligned}
$$

which is directly related to problem (2.4). The boundedness of the bilinear form (3.4) is obvious in view of the definition of the norms via (3.2) and is described in the following theorem.

THEOREM 3.2. There exists a constant $C_{b}$ independent of the parameters $\lambda, R_{p}^{-1}, \alpha_{p}$ such that for any $(\boldsymbol{u}, \boldsymbol{v}, p) \in \boldsymbol{U} \times \boldsymbol{V} \times P,(\boldsymbol{w}, \boldsymbol{z}, q) \in \boldsymbol{U} \times \boldsymbol{V} \times P$,

$$
|A((\boldsymbol{u}, \boldsymbol{v}, p),(\boldsymbol{w}, \boldsymbol{z}, q))| \leq C_{b}\left(\|\boldsymbol{u}\|_{\boldsymbol{U}}+\|\boldsymbol{v}\|_{\boldsymbol{V}}+\|p\|_{P}\right)\left(\|\boldsymbol{w}\|_{\boldsymbol{U}}+\|\boldsymbol{z}\|_{\boldsymbol{V}}+\|q\|_{P}\right) .
$$

The definiteness of the bilinear form, which can be expressed as

$$
\sup _{(\boldsymbol{u}, \boldsymbol{v}, p) \in \boldsymbol{U} \times \boldsymbol{V} \times P}|A((\boldsymbol{u}, \boldsymbol{v}, p),(\boldsymbol{w}, \boldsymbol{z}, q))|>0 \quad \text { for all } \begin{aligned}
& (\boldsymbol{u}, \boldsymbol{v}, p) \in \boldsymbol{U} \times \boldsymbol{V} \times P, \\
& (\boldsymbol{w}, \boldsymbol{z}, q) \neq(\mathbf{0}, \mathbf{0}, 0),
\end{aligned}
$$

is also easy to verify. Hence, according to the Banach-Necas-Babuska theorem (cf. [16]), problem (2.4) is well-posed if the bilinear form (3.4) satisfies the inf-sup condition (3.5) described in the following Theorem 3.3, which is our first main result.

THEOREM 3.3. There exists a constant $\beta>0$ independent of the parameters $\lambda, R_{p}^{-1}, \alpha_{p}$, such that

$$
\inf _{(\boldsymbol{u}, \boldsymbol{v}, p) \in \boldsymbol{U} \times \boldsymbol{V} \times P} \sup _{(\boldsymbol{w}, \boldsymbol{z}, q) \in \boldsymbol{U} \times \boldsymbol{V} \times P} \frac{A((\boldsymbol{u}, \boldsymbol{v}, p),(\boldsymbol{w}, \boldsymbol{z}, q))}{\left(\|\boldsymbol{u}\|_{\boldsymbol{U}}+\|\boldsymbol{v}\|_{\boldsymbol{V}}+\|p\|_{P}\right)\left(\|\boldsymbol{w}\|_{\boldsymbol{U}}+\|\boldsymbol{z}\|_{\boldsymbol{V}}+\|q\|_{P}\right)} \geq \beta .
$$

Proof. Case I:

$$
\rho=\min \left\{\lambda, R_{p}^{-1}\right\}=\lambda, \quad \text { hence } \quad \lambda \leq R_{p}^{-1}, \quad \gamma^{-1} \leq \rho=\lambda .
$$

For any $(\boldsymbol{u}, \boldsymbol{v}, p) \in \boldsymbol{U} \times \boldsymbol{V} \times P$, in view of the surjectivity of the divergence operator mapping $\boldsymbol{U}$ onto $P$, using Lemma 2.3, one finds that (cf. [9]) there exists

$$
\boldsymbol{u}_{0} \in \boldsymbol{U} \quad \text { such that } \quad \operatorname{div} \boldsymbol{u}_{0}=\frac{1}{\sqrt{\lambda}} p, \quad\left\|\boldsymbol{u}_{0}\right\|_{1} \leq \beta_{s}^{-1} \frac{1}{\sqrt{\lambda}}\|p\| .
$$


We choose

$$
\boldsymbol{w}=\delta \boldsymbol{u}-\frac{1}{\sqrt{\lambda}} \boldsymbol{u}_{0}, \quad \boldsymbol{z}=\delta \boldsymbol{v}, \quad q=-\delta p-\gamma^{-1} \operatorname{div} \boldsymbol{v},
$$

where $\delta$ is a positive constant that will be determined later.

First we verify the boundedness of $(\boldsymbol{w}, \boldsymbol{z}, q)$ by $(\boldsymbol{u}, \boldsymbol{v}, p)$. By (3.6) and noting that $\gamma^{-1} \leq \rho=\lambda$, we have

$$
\begin{aligned}
\left(\frac{1}{\sqrt{\lambda}} \boldsymbol{u}_{0}, \frac{1}{\sqrt{\lambda}} \boldsymbol{u}_{0}\right)_{\boldsymbol{U}} & =\left(\boldsymbol{\epsilon}\left(\frac{1}{\sqrt{\lambda}} \boldsymbol{u}_{0}\right), \boldsymbol{\epsilon}\left(\frac{1}{\sqrt{\lambda}} \boldsymbol{u}_{0}\right)\right)+\lambda\left(\operatorname{div} \frac{1}{\sqrt{\lambda}} \boldsymbol{u}_{0}, \operatorname{div} \frac{1}{\sqrt{\lambda}} \boldsymbol{u}_{0}\right) \\
& =\left(\frac{1}{\lambda} \boldsymbol{\epsilon}\left(\boldsymbol{u}_{0}\right), \boldsymbol{\epsilon}\left(\boldsymbol{u}_{0}\right)\right)+\left(\operatorname{div} \boldsymbol{u}_{0}, \operatorname{div} \boldsymbol{u}_{0}\right) \leq \frac{1}{\lambda} \beta_{s}^{-2} \frac{1}{\lambda}\|p\|^{2}+\frac{1}{\lambda}(p, p) \\
& \leq\left(\frac{1}{\lambda} \beta_{s}^{-2}+1\right) \frac{1}{\lambda}\|p\|^{2} \leq\left(\frac{1}{\lambda} \beta_{s}^{-2}+1\right) \gamma\|p\|^{2}=\left(\frac{1}{\lambda} \beta_{s}^{-2}+1\right)(p, p)_{P} .
\end{aligned}
$$

Next, since $1 \leq \lambda$, we get the boundedness of $\boldsymbol{w}$, i.e.,

$$
\|\boldsymbol{w}\|_{\boldsymbol{U}} \leq \delta\|\boldsymbol{u}\|_{\boldsymbol{U}}+\sqrt{\lambda^{-1} \beta_{s}^{-2}+1}\|p\|_{P} \leq \delta\|\boldsymbol{u}\|_{\boldsymbol{U}}+\sqrt{\beta_{s}^{-2}+1}\|p\|_{P} .
$$

It is obvious that $\|\boldsymbol{z}\|_{\boldsymbol{V}}=\delta\|\boldsymbol{v}\|_{\boldsymbol{V}}$. We still need to bound $q$. Using (3.7) and

$$
\left(\gamma^{-1} \operatorname{div} \boldsymbol{v}, \gamma^{-1} \operatorname{div} \boldsymbol{v}\right)_{P}=\gamma\left(\gamma^{-1} \operatorname{div} \boldsymbol{v}, \gamma^{-1} \operatorname{div} \boldsymbol{v}\right)=\gamma^{-1}(\operatorname{div} \boldsymbol{v}, \operatorname{div} \boldsymbol{v}) \leq(\boldsymbol{v}, \boldsymbol{v})_{\boldsymbol{V}},
$$

we obtain

$$
\|q\|_{P} \leq \delta\|p\|_{P}+\|\boldsymbol{v}\|_{\boldsymbol{V}}
$$

Putting the above estimates together we get

$$
\|\boldsymbol{w}\|_{\boldsymbol{U}}+\|\boldsymbol{z}\|_{\boldsymbol{V}}+\|q\|_{P} \leq\left(\delta+\sqrt{\beta_{s}^{-2}+1}\right)\left(\|\boldsymbol{u}\|_{\boldsymbol{U}}+\|\boldsymbol{v}\|_{\boldsymbol{V}}+\|p\|_{P}\right)
$$

and thus the boundedness of $(\boldsymbol{w}, \boldsymbol{z}, q)$ by $(\boldsymbol{u}, \boldsymbol{v}, p)$.

Next we show the coercivity of $A((\boldsymbol{u}, \boldsymbol{v}, p),(\boldsymbol{w}, \boldsymbol{z}, q))$. Using (3.7) and (3.6), we find

$$
\begin{aligned}
& A((\boldsymbol{u}, \boldsymbol{v}, p),(\boldsymbol{w}, \boldsymbol{z}, q)) \\
&=(\boldsymbol{\epsilon}(\boldsymbol{u}), \boldsymbol{\epsilon}(\boldsymbol{w}))+\lambda(\operatorname{div} \boldsymbol{u}, \operatorname{div} \boldsymbol{w})-(p, \operatorname{div} \boldsymbol{w})+R_{p}^{-1}(\boldsymbol{v}, \boldsymbol{z})-(p, \operatorname{div} \boldsymbol{z}) \\
& \quad-(\operatorname{div} \boldsymbol{u}, q)-(\operatorname{div} \boldsymbol{v}, q)-\alpha_{p}(p, q) \\
&=\left(\boldsymbol{\epsilon}(\boldsymbol{u}), \delta \boldsymbol{\epsilon}(\boldsymbol{u})-\frac{1}{\sqrt{\lambda}} \boldsymbol{\epsilon}\left(\boldsymbol{u}_{0}\right)\right)+\lambda\left(\operatorname{div} \boldsymbol{u}, \delta \operatorname{div} \boldsymbol{u}-\frac{1}{\sqrt{\lambda}} \operatorname{div} \boldsymbol{u}_{0}\right)+R_{p}^{-1}(\boldsymbol{v}, \delta \boldsymbol{v}) \\
& \quad-\left(p, \delta \operatorname{div} \boldsymbol{u}-\frac{1}{\sqrt{\lambda}} \operatorname{div} \boldsymbol{u}_{0}\right)-(p, \delta \operatorname{div} \boldsymbol{v})-\left(\operatorname{div} \boldsymbol{u},-\delta p-\gamma^{-1} \operatorname{div} \boldsymbol{v}\right) \\
& \quad-\left(\operatorname{div} \boldsymbol{v},-\delta p-\gamma^{-1} \operatorname{div} \boldsymbol{v}\right)-\alpha_{p}\left(p,-\delta p-\gamma^{-1} \operatorname{div} \boldsymbol{v}\right) \\
&=\delta(\boldsymbol{\epsilon}(\boldsymbol{u}), \boldsymbol{\epsilon}(\boldsymbol{u}))-\frac{1}{\sqrt{\lambda}}\left(\boldsymbol{\epsilon}(\boldsymbol{u}), \boldsymbol{\epsilon}\left(\boldsymbol{u}_{0}\right)\right)+\delta \lambda(\operatorname{div} \boldsymbol{u}, \operatorname{div} \boldsymbol{u})-\sqrt{\lambda}\left(\operatorname{div} \boldsymbol{u}, \operatorname{div} \boldsymbol{u}_{0}\right) \\
& \quad-\delta(p, \operatorname{div} \boldsymbol{u})+\frac{1}{\sqrt{\lambda}}\left(p, \operatorname{div} \boldsymbol{u}_{0}\right)+\delta R_{p}^{-1}(\boldsymbol{v}, \boldsymbol{v})-\delta(p, \operatorname{div} \boldsymbol{v}) \\
& \quad+\delta(\operatorname{div} \boldsymbol{u}, p)+\gamma^{-1}(\operatorname{div} \boldsymbol{u}, \operatorname{div} \boldsymbol{v})+\delta(\operatorname{div} \boldsymbol{v}, p) \\
& \quad+\gamma^{-1}(\operatorname{div} \boldsymbol{v}, \operatorname{div} \boldsymbol{v})+\delta \alpha_{p}(p, p)+\alpha_{p}\left(p, \gamma^{-1} \operatorname{div} \boldsymbol{v}\right)
\end{aligned}
$$




$$
\begin{aligned}
=_{\text {by }(3.6)} & \delta(\boldsymbol{\epsilon}(\boldsymbol{u}), \boldsymbol{\epsilon}(\boldsymbol{u}))-\frac{1}{\sqrt{\lambda}}\left(\boldsymbol{\epsilon}(\boldsymbol{u}), \boldsymbol{\epsilon}\left(\boldsymbol{u}_{0}\right)\right)+\delta \lambda(\operatorname{div} \boldsymbol{u}, \operatorname{div} \boldsymbol{u})-(\operatorname{div} \boldsymbol{u}, p) \\
+ & \frac{1}{\lambda}(p, p)+\delta R_{p}^{-1}(\boldsymbol{v}, \boldsymbol{v})+\gamma^{-1}(\operatorname{div} \boldsymbol{u}, \operatorname{div} \boldsymbol{v}) \\
+ & \gamma^{-1}(\operatorname{div} \boldsymbol{v}, \operatorname{div} \boldsymbol{v})+\delta \alpha_{p}(p, p)+\alpha_{p}\left(p, \gamma^{-1} \operatorname{div} \boldsymbol{v}\right) .
\end{aligned}
$$

Applying Young's inequality and using (3.6), we therefore obtain

$$
\begin{aligned}
& A((\boldsymbol{u}, \boldsymbol{v}, p),(\boldsymbol{w}, \boldsymbol{z}, q)) \\
& =\delta(\boldsymbol{\epsilon}(\boldsymbol{u}), \boldsymbol{\epsilon}(\boldsymbol{u}))-\frac{1}{\sqrt{\lambda}}\left(\boldsymbol{\epsilon}(\boldsymbol{u}), \boldsymbol{\epsilon}\left(\boldsymbol{u}_{0}\right)\right)+\delta \lambda(\operatorname{div} \boldsymbol{u}, \operatorname{div} \boldsymbol{u})-(\operatorname{div} \boldsymbol{u}, p)+\frac{1}{\lambda}(p, p) \\
& \quad+\delta R_{p}^{-1}(\boldsymbol{v}, \boldsymbol{v})+\gamma^{-1}(\operatorname{div} \boldsymbol{u}, \operatorname{div} \boldsymbol{v})+\gamma^{-1}(\operatorname{div} \boldsymbol{v}, \operatorname{div} \boldsymbol{v}) \\
& \quad+\delta \alpha_{p}(p, p)+\alpha_{p}\left(p, \gamma^{-1} \operatorname{div} \boldsymbol{v}\right) \\
& \geq \delta(\boldsymbol{\epsilon}(\boldsymbol{u}), \boldsymbol{\epsilon}(\boldsymbol{u}))-\frac{1}{2} \frac{1}{\sqrt{\lambda}} \epsilon_{1}^{-1}(\boldsymbol{\epsilon}(\boldsymbol{u}), \boldsymbol{\epsilon}(\boldsymbol{u}))-\frac{1}{2} \frac{1}{\sqrt{\lambda}} \epsilon_{1}\left(\boldsymbol{\epsilon}\left(\boldsymbol{u}_{0}\right), \boldsymbol{\epsilon}\left(\boldsymbol{u}_{0}\right)\right)+\delta \lambda(\operatorname{div} \boldsymbol{u}, \operatorname{div} \boldsymbol{u}) \\
& \quad-\frac{1}{2} \epsilon_{2}^{-1} \lambda(\operatorname{div} \boldsymbol{u}, \operatorname{div} \boldsymbol{u})-\frac{1}{2} \epsilon_{2} \frac{1}{\lambda}(p, p)+\frac{1}{\lambda}(p, p)+\delta R_{p}^{-1}(\boldsymbol{v}, \boldsymbol{v}) \\
& \quad-\frac{1}{2} \epsilon_{3}^{-1} \gamma^{-1}(\operatorname{div} \boldsymbol{u}, \operatorname{div} \boldsymbol{u})-\frac{1}{2} \epsilon_{3} \gamma^{-1}(\operatorname{div} \boldsymbol{v}, \operatorname{div} \boldsymbol{v})+\gamma^{-1}(\operatorname{div} \boldsymbol{v}, \operatorname{div} \boldsymbol{v}) \\
& \quad+\delta \alpha_{p}(p, p)-\frac{1}{2} \epsilon_{4} \gamma^{-1}(\operatorname{div} \boldsymbol{v}, \operatorname{div} \boldsymbol{v})-\frac{1}{2} \epsilon_{4}^{-1} \alpha_{p}^{2} \gamma^{-1}(p, p) \\
& \geq{ }_{\text {by }(3.6)}\left(\delta-\frac{1}{2} \frac{1}{\sqrt{\lambda}} \epsilon_{1}^{-1}\right)(\boldsymbol{\epsilon}(\boldsymbol{u}), \boldsymbol{\epsilon}(\boldsymbol{u}))+\left(\delta-\frac{1}{2} \epsilon_{2}^{-1}\right) \lambda(\operatorname{div} \boldsymbol{u}, \operatorname{div} \boldsymbol{u}) \\
& \quad-\frac{1}{2} \epsilon_{3}^{-1} \gamma^{-1}(\operatorname{div} \boldsymbol{u}, \operatorname{div} \boldsymbol{u})+\delta R_{p}^{-1}(\boldsymbol{v}, \boldsymbol{v})+\left(1-\frac{1}{2} \epsilon_{3}-\frac{1}{2} \epsilon_{4}\right) \gamma^{-1}(\operatorname{div} \boldsymbol{v}, \operatorname{div} \boldsymbol{v}) \\
& +\left(1-\frac{1}{2} \frac{1}{\sqrt{\lambda}} \epsilon_{1} \beta_{s}^{-2}-\frac{1}{2} \epsilon_{2}\right) \frac{1}{\lambda}(p, p)+\left(\delta-\frac{1}{2} \epsilon_{4}^{-1} \alpha_{p} \gamma^{-1}\right) \alpha_{p}(p, p) .
\end{aligned}
$$

Now, letting $\epsilon_{1}=\frac{\beta_{s}^{2}}{2}, \epsilon_{2}=\epsilon_{3}=\epsilon_{4}=\frac{1}{2}$ and noting that $\rho^{-1} \leq \gamma, \lambda \geq \gamma^{-1}>0$, and $\lambda \geq 1$, we further conclude that

$$
\begin{aligned}
& A((\boldsymbol{u}, \boldsymbol{v}, p),(\boldsymbol{w}, \boldsymbol{z}, q)) \\
& \geq\left(\delta-\frac{1}{\sqrt{\lambda}} \beta_{s}^{-2}\right)(\boldsymbol{\epsilon}(\boldsymbol{u}), \boldsymbol{\epsilon}(\boldsymbol{u}))+(\delta-2) \lambda(\operatorname{div} \boldsymbol{u}, \operatorname{div} \boldsymbol{u})+\delta R_{p}^{-1}(\boldsymbol{v}, \boldsymbol{v}) \\
& \quad+\frac{1}{2} \gamma^{-1}(\operatorname{div} \boldsymbol{v}, \operatorname{div} \boldsymbol{v})+\frac{1}{2} \frac{1}{\lambda}(p, p)+\left(\delta-\alpha_{p} \gamma^{-1}\right) \alpha_{p}(p, p) .
\end{aligned}
$$

Next, letting $\delta:=\max \left\{\beta_{s}^{-2}+\frac{1}{2}, 2+\frac{1}{2}\right\}$ and noting that $\alpha_{p} \leq \gamma, \lambda \geq 1$, we arrive at the following coercivity estimate

$$
\begin{aligned}
A((\boldsymbol{u}, \boldsymbol{v}, p),(\boldsymbol{w}, \boldsymbol{z}, q)) \geq & \frac{1}{2}(\boldsymbol{\epsilon}(\boldsymbol{u}), \boldsymbol{\epsilon}(\boldsymbol{u}))+\frac{1}{2} \lambda(\operatorname{div} \boldsymbol{u}, \operatorname{div} \boldsymbol{u})+\frac{1}{2} R_{p}^{-1}(\boldsymbol{v}, \boldsymbol{v}) \\
& +\frac{1}{2} \gamma^{-1}(\operatorname{div} \boldsymbol{v}, \operatorname{div} \boldsymbol{v})+\frac{1}{2} \frac{1}{\lambda}(p, p)+\alpha_{p}(p, p) \\
\geq & \frac{1}{2}\left(\|\boldsymbol{u}\|_{\boldsymbol{U}}^{2}+\|\boldsymbol{v}\|_{\boldsymbol{V}}^{2}+\|p\|_{P}^{2}\right) .
\end{aligned}
$$

Case II:

$$
\rho=\min \left\{\lambda, R_{p}^{-1}\right\}=R_{p}^{-1}, \quad \text { hence } \quad \lambda \geq R_{p}^{-1}, \quad \gamma^{-1} \leq \rho=R_{p}^{-1}
$$


For any $(\boldsymbol{u}, \boldsymbol{v}, p) \in \boldsymbol{U} \times \boldsymbol{V} \times P$, in view of the surjectivity of the divergence operator mapping $\boldsymbol{V}$ onto $P$, using Lemma 2.2, one finds that (cf. [9]) there exists

$$
\boldsymbol{v}_{0} \in \boldsymbol{V} \quad \text { such that } \quad \operatorname{div} \boldsymbol{v}_{0}=\sqrt{R_{p}} p, \quad\left\|\boldsymbol{v}_{0}\right\|_{\text {div }} \leq \beta_{d}^{-1} \sqrt{R_{p}}\|p\| .
$$

We choose

$$
\boldsymbol{w}=\delta \boldsymbol{u}, \quad \boldsymbol{z}=\delta \boldsymbol{v}-\sqrt{R_{p}} \boldsymbol{v}_{0}, \quad q=-\delta p-\gamma^{-1} \operatorname{div} \boldsymbol{v}
$$

where $\delta$ is a constant which we will specify later.

Again we verify the boundedness of $(\boldsymbol{w}, \boldsymbol{z}, q)$ by $(\boldsymbol{u}, \boldsymbol{v}, p)$ first. It is obvious that $\|\boldsymbol{w}\|_{U}=\delta\|\boldsymbol{u}\|_{\boldsymbol{U}}$. Moreover, by (3.10) and noting that $\gamma^{-1} \leq \rho=R_{p}^{-1}$, we have

$$
\begin{gathered}
\left(\sqrt{R}_{p} \boldsymbol{v}_{0}, \sqrt{R}_{p} \boldsymbol{v}_{0}\right)_{\boldsymbol{V}}=R_{p}^{-1}\left(\sqrt{R}_{p} \boldsymbol{v}_{0}, \sqrt{R}_{p} \boldsymbol{v}_{0}\right)+\gamma^{-1}\left(\operatorname{div}\left(\sqrt{R_{p}} \boldsymbol{v}_{0}\right), \operatorname{div}\left(\sqrt{R_{p}} \boldsymbol{v}_{0}\right)\right) \\
\leq\left(\boldsymbol{v}_{0}, \boldsymbol{v}_{0}\right)+\left(\operatorname{div} \boldsymbol{v}_{0}, \operatorname{div} \boldsymbol{v}_{0}\right) \leq \beta_{d}^{-2} R_{p}\|p\|^{2} \leq \beta_{d}^{-2} \gamma\|p\|^{2} \leq \beta_{d}^{-2}(p, p)_{P}
\end{gathered}
$$

Hence, we get the boundedness of $z$, i.e.,

$$
\|\boldsymbol{z}\|_{\boldsymbol{V}} \leq \delta\|\boldsymbol{v}\|_{\boldsymbol{V}}+\beta_{d}^{-1}\|p\|_{P}
$$

The boundedness of $q$ follows as in (3.8).

Next we verify the coercivity of $A((\boldsymbol{u}, \boldsymbol{v}, p),(\boldsymbol{w}, \boldsymbol{z}, q))$ in Case II. Using the definition of $(\boldsymbol{w}, \boldsymbol{z}, q)$ and (3.9), we find

$$
\begin{aligned}
& A((\boldsymbol{u}, \boldsymbol{v}, p),(\boldsymbol{w}, \boldsymbol{z}, q)) \\
&=(\boldsymbol{\epsilon}(\boldsymbol{u}), \boldsymbol{\epsilon}(\boldsymbol{w}))+\lambda(\operatorname{div} \boldsymbol{u}, \operatorname{div} \boldsymbol{w})-(p, \operatorname{div} \boldsymbol{w})+R_{p}^{-1}(\boldsymbol{v}, \boldsymbol{z})-(p, \operatorname{div} \boldsymbol{z}) \\
& \quad-(\operatorname{div} \boldsymbol{u}, q)-(\operatorname{div} \boldsymbol{v}, q)-\alpha_{p}(p, q) \\
&=(\boldsymbol{\epsilon}(\boldsymbol{u}), \delta \boldsymbol{\epsilon}(\boldsymbol{u}))+\lambda(\operatorname{div} \boldsymbol{u}, \delta \operatorname{div} \boldsymbol{u})-(p, \delta \operatorname{div} \boldsymbol{u})+R_{p}^{-1}\left(\boldsymbol{v}, \delta \boldsymbol{v}-\sqrt{R_{p}} \boldsymbol{v}_{0}\right) \\
& \quad-\left(p, \delta \operatorname{div} \boldsymbol{v}-\sqrt{R_{p}} \operatorname{div} \boldsymbol{v}_{0}\right)-\left(\operatorname{div} \boldsymbol{u},-\delta p-\gamma^{-1} \operatorname{div} \boldsymbol{v}\right) \\
& \quad-\left(\operatorname{div} \boldsymbol{v},-\delta p-\gamma^{-1} \operatorname{div} \boldsymbol{v}\right)-\alpha_{p}\left(p,-\delta p-\gamma^{-1} \operatorname{div} \boldsymbol{v}\right) \\
&=\delta(\boldsymbol{\epsilon}(\boldsymbol{u}), \boldsymbol{\epsilon}(\boldsymbol{u}))+\delta \lambda(\operatorname{div} \boldsymbol{u}, \operatorname{div} \boldsymbol{u})-\delta(p, \operatorname{div} \boldsymbol{u})+\delta R_{p}^{-1}(\boldsymbol{v}, \boldsymbol{v})-\left(R_{p}^{-1 / 2} \boldsymbol{v}, \boldsymbol{v}_{0}\right) \\
& \quad-\delta(p, \operatorname{div} \boldsymbol{v})+\left(p, \sqrt{R_{p}} \operatorname{div} \boldsymbol{v}_{0}\right)+\delta(\operatorname{div} \boldsymbol{u}, p)+\gamma^{-1}(\operatorname{div} \boldsymbol{u}, \operatorname{div} \boldsymbol{v}) \\
& \quad+\delta(\operatorname{div} \boldsymbol{v}, p)+\gamma^{-1}(\operatorname{div} \boldsymbol{v}, \operatorname{div} \boldsymbol{v})+\delta \alpha_{p}(p, p)+\alpha_{p}\left(p, \gamma^{-1} \operatorname{div} \boldsymbol{v}\right) \\
&=\delta(\boldsymbol{\epsilon}(\boldsymbol{u}), \boldsymbol{\epsilon}(\boldsymbol{u}))+\delta \lambda(\operatorname{div} \boldsymbol{u}, \operatorname{div} \boldsymbol{u})+\delta R_{p}^{-1}(\boldsymbol{v}, \boldsymbol{v})-\left(R_{p}^{-1 / 2} \boldsymbol{v}, \boldsymbol{v}_{0}\right) \\
& \quad+\left(p, \sqrt{R_{p}} \operatorname{div} \boldsymbol{v}_{0}\right)+\gamma^{-1}(\operatorname{div} \boldsymbol{u}, \operatorname{div} \boldsymbol{v}) \\
& \quad+\gamma^{-1}(\operatorname{div} \boldsymbol{v}, \operatorname{div} \boldsymbol{v})+\delta \alpha_{p}(p, p)+\alpha_{p}\left(p, \gamma^{-1} \operatorname{div} \boldsymbol{v}\right) .
\end{aligned}
$$

Applying Young's inequality and using (3.9), we get

$$
\begin{aligned}
& A((\boldsymbol{u}, \boldsymbol{v}, p),(\boldsymbol{w}, \boldsymbol{z}, q)) \\
& =\delta(\boldsymbol{\epsilon}(\boldsymbol{u}), \boldsymbol{\epsilon}(\boldsymbol{u}))+\delta \lambda(\operatorname{div} \boldsymbol{u}, \operatorname{div} \boldsymbol{u})+\delta R_{p}^{-1}(\boldsymbol{v}, \boldsymbol{v})-\left(R_{p}^{-1 / 2} \boldsymbol{v}, \boldsymbol{v}_{0}\right) \\
& \quad+\left(p, \sqrt{R_{p}} \operatorname{div} \boldsymbol{v}_{0}\right)+\gamma^{-1}(\operatorname{div} \boldsymbol{u}, \operatorname{div} \boldsymbol{v})+\gamma^{-1}(\operatorname{div} \boldsymbol{v}, \operatorname{div} \boldsymbol{v}) \\
& \quad+\delta \alpha_{p}(p, p)+\alpha_{p}\left(p, \gamma^{-1} \operatorname{div} \boldsymbol{v}\right)
\end{aligned}
$$




$$
\begin{aligned}
& \geq \delta(\boldsymbol{\epsilon}(\boldsymbol{u}), \boldsymbol{\epsilon}(\boldsymbol{u}))+\delta \lambda(\operatorname{div} \boldsymbol{u}, \operatorname{div} \boldsymbol{u})+\delta R_{p}^{-1}(\boldsymbol{v}, \boldsymbol{v})-\frac{1}{2} \epsilon_{1}^{-1} R_{p}^{-1}(\boldsymbol{v}, \boldsymbol{v})-\frac{1}{2} \epsilon_{1}\left(\boldsymbol{v}_{0}, \boldsymbol{v}_{0}\right) \\
& \quad+R_{p}(p, p)-\frac{1}{2} \epsilon_{2}^{-1} \gamma^{-1}(\operatorname{div} \boldsymbol{u}, \operatorname{div} \boldsymbol{u})-\frac{1}{2} \epsilon_{2} \gamma^{-1}(\operatorname{div} \boldsymbol{v}, \operatorname{div} \boldsymbol{v}) \\
& \quad+\gamma^{-1}(\operatorname{div} \boldsymbol{v}, \operatorname{div} \boldsymbol{v})+\delta \alpha_{p}(p, p)-\frac{1}{2} \epsilon_{4} \gamma^{-1}(\operatorname{div} \boldsymbol{v}, \operatorname{div} \boldsymbol{v})-\frac{1}{2} \epsilon_{4}^{-1} \alpha_{p}^{2} \gamma^{-1}(p, p) \\
& \geq \delta(\boldsymbol{\epsilon}(\boldsymbol{u}), \boldsymbol{\epsilon}(\boldsymbol{u}))+\delta \lambda(\operatorname{div} \boldsymbol{u}, \operatorname{div} \boldsymbol{u})-\frac{1}{2} \epsilon_{2}^{-1} \gamma^{-1}(\operatorname{div} \boldsymbol{u}, \operatorname{div} \boldsymbol{u}) \\
& \quad+\left(\delta-\frac{1}{2} \epsilon_{1}^{-1}\right) R_{p}^{-1}(\boldsymbol{v}, \boldsymbol{v})+\left(1-\frac{1}{2} \epsilon_{2}-\frac{1}{2} \epsilon_{4}\right) \gamma^{-1}(\operatorname{div} \boldsymbol{v}, \operatorname{div} \boldsymbol{v}) \\
& \quad+\left(1-\frac{1}{2} \epsilon_{1} \beta_{d}^{-2}\right) R_{p}(p, p)+\left(\delta-\frac{1}{2} \epsilon_{4}^{-1} \alpha_{p} \gamma^{-1}\right) \alpha_{p}(p, p) .
\end{aligned}
$$

Now, letting $\epsilon_{1}=\beta_{d}^{2}, \epsilon_{2}=\epsilon_{4}=\frac{1}{2}$ and noting that $\rho^{-1} \leq \gamma, \lambda \geq \rho \geq \gamma^{-1}>0$ it follows that $A((\boldsymbol{u}, \boldsymbol{v}, p),(\boldsymbol{w}, \boldsymbol{z}, q)) \geq \delta(\boldsymbol{\epsilon}(\boldsymbol{u}), \boldsymbol{\epsilon}(\boldsymbol{u}))+(\delta-1) \lambda(\operatorname{div} \boldsymbol{u}, \operatorname{div} \boldsymbol{u})+\left(\delta-\frac{1}{2} \beta_{d}^{-2}\right) R_{p}^{-1}(\boldsymbol{v}, \boldsymbol{v})$

$$
+\frac{1}{2} \gamma^{-1}(\operatorname{div} \boldsymbol{v}, \operatorname{div} \boldsymbol{v})+\frac{1}{2} R_{p}(p, p)+\left(\delta-\alpha_{p} \gamma^{-1}\right) \alpha_{p}(p, p) .
$$

Next, we set $\delta=\max \left\{\frac{1}{2} \beta_{d}^{-2}+\frac{1}{2}, 1+\frac{1}{2}\right\}$, observe that $\alpha_{p} \leq \gamma$, and finally obtain the coercivity estimate

$$
\begin{aligned}
A((\boldsymbol{u}, \boldsymbol{v}, p),(\boldsymbol{w}, \boldsymbol{z}, q)) \geq & \frac{1}{2}(\boldsymbol{\epsilon}(\boldsymbol{u}), \boldsymbol{\epsilon}(\boldsymbol{u}))+\frac{1}{2} \lambda(\operatorname{div} \boldsymbol{u}, \operatorname{div} \boldsymbol{u})+\frac{1}{2} R_{p}^{-1}(\boldsymbol{v}, \boldsymbol{v}) \\
& +\frac{1}{2} \gamma^{-1}(\operatorname{div} \boldsymbol{v}, \operatorname{div} \boldsymbol{v})+\frac{1}{2} R_{p}(p, p)+\frac{1}{2} \alpha_{p}(p, p) \\
& \geq \frac{1}{2}\left(\|\boldsymbol{u}\|_{\boldsymbol{U}}^{2}+\|\boldsymbol{v}\|_{\boldsymbol{V}}^{2}+\|p\|_{P}^{2}\right),
\end{aligned}
$$

which completes the proof.

REMARK 3.4. The distinction of the two cases in the proof of Theorem 3.3 (and later on in the proof of Theorem 4.4) is due to the specific choice of the combined parameter-dependent norm induced by the inner products (3.2). In order to obtain a parameter-robust inf-sup condition in a norm providing also uniform boundedness of the bilinear form $A((\cdot, \cdot, \cdot),(\cdot, \cdot, \cdot))$ it would be sufficient to consider Case I, i.e., to use the combined norm

$$
\begin{aligned}
& (\boldsymbol{\epsilon}(\boldsymbol{u}), \boldsymbol{\epsilon}(\boldsymbol{u}))+\lambda(\operatorname{div} \boldsymbol{u}, \operatorname{div} \boldsymbol{u})+R_{p}^{-1}(\boldsymbol{v}, \boldsymbol{v}) \\
& \left.+\min \left\{\lambda, \alpha_{p}^{-1}\right\}(\operatorname{div} \boldsymbol{v}, \operatorname{div} \boldsymbol{v})+\max \left\{\lambda^{-1}, \alpha_{p}\right\}(p, p)\right)^{1 / 2} \\
& \approx(\boldsymbol{\epsilon}(\boldsymbol{u}), \boldsymbol{\epsilon}(\boldsymbol{u}))+\lambda(\operatorname{div} \boldsymbol{u}, \operatorname{div} \boldsymbol{u})+R_{p}^{-1}(\boldsymbol{v}, \boldsymbol{v}) \\
& \left.+\left(\frac{1}{\lambda}+\alpha_{p}\right)^{-1}(\operatorname{div} \boldsymbol{v}, \operatorname{div} \boldsymbol{v})+\left(\frac{1}{\lambda}+\alpha_{p}\right)(p, p)\right)^{1 / 2}
\end{aligned}
$$

for all $(\boldsymbol{u}, \boldsymbol{v}, p) \in \boldsymbol{U} \times \boldsymbol{V} \times P$. The reason for introducing the norm in the more general setting, which results in the two cases, i.e., two different definitions of the combined norm depending on which parameter defines $\rho$ and which one $\gamma$, is to obtain a norm on the product 
space that results in a tighter and physically sound stability estimate as given by the following Corollary 3.5 and an error estimate, see Theorem 5.2, over the whole parameter ranges.

The above theorem implies the following stability estimate.

COROLlARY 3.5. Let $(\boldsymbol{u}, \boldsymbol{v}, p) \in \boldsymbol{U} \times \boldsymbol{V} \times P$ be the solution of (2.4). Then there holds the estimate

$$
\|\boldsymbol{u}\|_{\boldsymbol{U}}+\|\boldsymbol{v}\|_{\boldsymbol{V}}+\|p\|_{P} \leq C_{1}\left(\|\boldsymbol{f}\|_{\boldsymbol{U}^{*}}+\|g\|_{P^{*}}\right),
$$

where $C_{1}$ is a constant independent of $\lambda, R_{p}^{-1}, \alpha_{p}$, and

$$
\|\boldsymbol{f}\|_{\boldsymbol{U}^{*}}=\sup _{\boldsymbol{w} \in \boldsymbol{U}} \frac{(\boldsymbol{f}, \boldsymbol{w})}{\|\boldsymbol{w}\|_{\boldsymbol{U}}},\|g\|_{P^{*}}=\sup _{q \in P} \frac{(g, q)}{\|q\|_{P}}=\gamma^{-1}\|g\| .
$$

REMARK 3.6. We want to emphasize that the parameter ranges as specified in (3.1) are indeed relevant since the variations of the model parameters are quite large in many applications. For that reason, Theorem 3.2 and Theorem 3.3 are very important and basic results that provide the parameter-robust stability of the model (2.4).

REMARK 3.7. Define

$$
\mathcal{B}:=\left[\begin{array}{ccc}
(-\operatorname{div} \epsilon-\lambda \nabla \operatorname{div})^{-1} & 0 & 0 \\
0 & \left(R_{p}^{-1} I+\gamma^{-1} \nabla \operatorname{div}\right)^{-1} & 0 \\
0 & 0 & (\gamma I)^{-1}
\end{array}\right] .
$$

Due to the theory presented in [29], Theorems 3.2 and 3.3 imply that the operator $\mathcal{B}$ in (3.11) defines a norm-equivalent (canonical) block-diagonal preconditioner for the operator $\mathcal{A}$ in (2.3) which is robust in all model parameters.

REMARK 3.8. Note that if $\lambda \ll R_{p}^{-1}$ and $0 \leq \alpha_{p} \leq \frac{1}{\lambda}$, then $\rho=\min \left\{\lambda, R_{p}^{-1}\right\}=\lambda$ and the norms defined in (3.2) are given by

$$
\begin{aligned}
(\boldsymbol{u}, \boldsymbol{w})_{\boldsymbol{U}} & =(\boldsymbol{\epsilon}(\boldsymbol{u}), \boldsymbol{\epsilon}(\boldsymbol{w}))+\lambda(\operatorname{div} \boldsymbol{u}, \operatorname{div} \boldsymbol{w}) \\
(\boldsymbol{v}, \boldsymbol{z})_{\boldsymbol{V}} & =R_{p}^{-1}(\boldsymbol{v}, \boldsymbol{z})+\lambda(\operatorname{div} \boldsymbol{v}, \operatorname{div} \boldsymbol{z}) \\
(p, q)_{P} & =\lambda^{-1}(p, q)
\end{aligned}
$$

Then the coercivity of $(\boldsymbol{\epsilon}(\boldsymbol{u}), \boldsymbol{\epsilon}(\boldsymbol{u}))+\lambda(\operatorname{div} \boldsymbol{u}, \operatorname{div} \boldsymbol{u})+R_{p}^{-1}(\boldsymbol{v}, \boldsymbol{v})$ on the kernel set

$$
\boldsymbol{Z}=\{(\boldsymbol{u}, \boldsymbol{v}) \in \boldsymbol{U} \times \boldsymbol{V}:(\operatorname{div} \boldsymbol{u}, q)+(\operatorname{div} \boldsymbol{v}, q)=0, \forall q \in P\}
$$

can be verified by direct computation. However, in this case the $H$ (div) inf-sup condition

$$
\inf _{q \in P} \sup _{\boldsymbol{v} \in \boldsymbol{V}} \frac{(\operatorname{div} \boldsymbol{v}, q)}{\|\boldsymbol{v}\|_{\boldsymbol{V}}\|q\|_{P}} \geq \beta_{v}>0
$$

fails if $R_{p}^{-1} \gg \lambda$, for instance, $\lambda \approx 1$ and $R_{p}^{-1} \gg 1$.

Hence, in order to obtain the inf-sup condition for $(\operatorname{div} \boldsymbol{u}, q)+(\operatorname{div} \boldsymbol{v}, q)$, namely,

$$
\inf _{q \in P} \sup _{(\boldsymbol{u}, \boldsymbol{v}) \in \boldsymbol{U} \times \boldsymbol{V}} \frac{(\operatorname{div} \boldsymbol{u}, q)+(\operatorname{div} \boldsymbol{v}, q)}{\left(\|\boldsymbol{u}\|_{\boldsymbol{U}}+\|\boldsymbol{v}\|_{\boldsymbol{V}}\right)\|q\|_{P}} \geq \beta_{s u}>0,
$$

the Stokes-type inf-sup condition from Lemma 2.3 has to be satisfied at the discrete level as we can see by choosing $(\boldsymbol{u}, \boldsymbol{v})=(\boldsymbol{u}, 0) \in \boldsymbol{U} \times \boldsymbol{V}$ in (3.12).

From the above observation, we conclude that we need to choose a proper space for the approximation of the displacement field $\boldsymbol{u}$, even if $\lambda$ is small, in order to satisfy the Stokes-type 
inf-sup condition stated in Lemma 2.3 at the discrete level. This shows that the discretization of (2.4) based on the spaces $P_{1} \times R T_{0} \times P_{0}$ cannot be uniformly stable with respect to all model parameters.

Therefore, in this work, we use the proper $H(\operatorname{div})$ conforming spaces to approximate the displacement field as well as the Darcy velocity. In the right combination and with matching approximation orders, the corresponding mixed finite element methods then allow to satisfy the divergence condition pointwise, resulting in strong mass conservation. Details will be presented in the following Section 4.

4. Uniformly stable and strongly mass conservative discretizations. There are various discretizations that meet the requirements for the proof of the full parameter-robust stability that will be presented in this section. They include conforming as well as nonconforming methods. In general, whenever $U_{h} / P_{h}$ is a Stokes-stable pair and $V_{h} / P_{h}$ satisfies the $H$ (div) inf-sup condition similar to (4.7), the norm that we have proposed in Section 3 allows to prove the full parameter-robust stability using similar arguments as in the proof of Theorem 4.4. To give a few examples, the triplets $C R_{l} / R T_{l-1} / P_{l-1}(l=1,2)$ together with stabilization $[17,20]$ does result in a parameter-robust stable discretization of the Biot model if the norms are defined as in Section 3. The same is true for the conforming discretizations based on the spaces $P_{2} / R T_{0} / P_{0}$ (in 2D), $P_{2}^{s t a b} / R T_{0} / P_{0}$ (in 3D), or $P_{2} / R T_{1} / P_{1}$. However, these finite element methods do not have the property of strong mass conservation in the sense of Proposition 4.2 although they result in parameter-robust inf-sup stability under the norms we proposed in Section 3.

In recent years, discontinuous Galerkin methods have been developed to solve various problems $[2,3,12,13,21]$. In this section, motivated by the works [22, 23, 34], we propose discretizations of the Biot's model problem (2.4). These discretizations preserve the divergence condition (namely, the last equation in (2.2)) pointwise, which results in a strong conservation of mass; see Proposition 4.2. Furthermore, they are also locking-free when the Lamé parameter $\lambda$ tends to infinity. First we introduce some notation.

4.1. Preliminaries and notation. By $\mathcal{T}_{h}$ we denote a shape-regular triangulation of mesh-size $h$ of the domain $\Omega$ into triangles $\{K\}$. We further denote by $\mathcal{E}_{h}^{I}$ the set of all interior edges (or faces) of $\mathcal{T}_{h}$ and by $\mathcal{E}_{h}^{B}$ the set of all boundary edges (or faces); we set $\mathcal{E}_{h}=\mathcal{E}_{h}^{I} \cup \mathcal{E}_{h}^{B}$.

For $s \geq 1$, we define

$$
H^{s}\left(\mathcal{T}_{h}\right)=\left\{\phi \in L^{2}(\Omega), \text { such that }\left.\phi\right|_{K} \in H^{s}(K) \text { for all } K \in \mathcal{T}_{h}\right\}
$$

The vector functions are represented column-wise.

As we consider discontinuous Galerkin (DG) discretizations, we define some trace operators next. Let $e=\partial K_{1} \cap \partial K_{2}$ be the common boundary (interface) of two subdomains $K_{1}$ and $K_{2}$ in $\mathcal{T}_{h}$, and $\boldsymbol{n}_{1}$ and $\boldsymbol{n}_{2}$ be unit normal vectors to $e$ pointing to the exterior of $K_{1}$ and $K_{2}$, respectively. For any edge (or face) $e \in \mathcal{E}_{h}^{I}$ and a scalar $q \in H^{1}\left(\mathcal{T}_{h}\right.$ ), a vector $\boldsymbol{v} \in H^{1}\left(\mathcal{T}_{h}\right)^{d}$, and a tensor $\boldsymbol{\tau} \in H^{1}\left(\mathcal{T}_{h}\right)^{d \times d}$, we define the averages

$$
\{\boldsymbol{v}\}=\frac{1}{2}\left(\left.\boldsymbol{v}\right|_{\partial K_{1} \cap e} \cdot \boldsymbol{n}_{1}-\left.\boldsymbol{v}\right|_{\partial K_{2} \cap e} \cdot \boldsymbol{n}_{2}\right), \quad\{\boldsymbol{\tau}\}=\frac{1}{2}\left(\left.\boldsymbol{\tau}\right|_{\partial K_{1} \cap e} \boldsymbol{n}_{1}-\left.\boldsymbol{\tau}\right|_{\partial K_{2} \cap e} \boldsymbol{n}_{2}\right)
$$

and jumps

$[q]=\left.q\right|_{\partial K_{1} \cap e}-\left.q\right|_{\partial K_{2} \cap e}, \quad[\boldsymbol{v}]=\left.\boldsymbol{v}\right|_{\partial K_{1} \cap e}-\left.\boldsymbol{v}\right|_{\partial K_{2} \cap e}, \quad \llbracket \boldsymbol{v} \rrbracket=\left.\boldsymbol{v}\right|_{\partial K_{1} \cap e} \odot \boldsymbol{n}_{1}+\left.\boldsymbol{v}\right|_{\partial K_{2} \cap e} \odot \boldsymbol{n}_{2}$,

where $\boldsymbol{v} \odot \boldsymbol{n}=\frac{1}{2}\left(\boldsymbol{v} \boldsymbol{n}^{T}+\boldsymbol{n} \boldsymbol{v}^{T}\right)$ is the symmetric part of the tensor product of $\boldsymbol{v}$ and $\boldsymbol{n}$.

When $e \in \mathcal{E}_{h}^{B}$, then the above quantities are defined as

$$
\{\boldsymbol{v}\}=\left.\boldsymbol{v}\right|_{e} \cdot \boldsymbol{n}, \quad\{\boldsymbol{\tau}\}=\left.\boldsymbol{\tau}\right|_{e} \boldsymbol{n}, \quad[q]=\left.q\right|_{e}, \quad[\boldsymbol{v}]=\left.\boldsymbol{v}\right|_{e}, \quad \llbracket \boldsymbol{v} \rrbracket=\left.\boldsymbol{v}\right|_{e} \odot \boldsymbol{n} .
$$


If $\boldsymbol{n}_{K}$ is the outward unit normal to $\partial K$, then it is easy to verify that $\sum_{K \in \mathcal{T}_{h}} \int_{\partial K} \boldsymbol{v} \cdot \boldsymbol{n}_{K} q d s=\sum_{e \in \mathcal{E}_{h}} \int_{e}\{\boldsymbol{v}\}[q] d s, \quad$ for all $\boldsymbol{v} \in H(\operatorname{div} ; \Omega), \quad$ for all $q \in H^{1}\left(\mathcal{T}_{h}\right)$.

Also, for $\boldsymbol{\tau} \in H^{1}(\Omega)^{d \times d}$ and for all $\boldsymbol{v} \in H^{1}\left(\mathcal{T}_{h}\right)^{d}$, we have

$$
\sum_{K \in \mathcal{T}_{h}} \int_{\partial K}\left(\boldsymbol{\tau} \boldsymbol{n}_{K}\right) \cdot \boldsymbol{v} d s=\sum_{e \in \mathcal{E}_{h}} \int_{e}\{\boldsymbol{\tau}\} \cdot[\boldsymbol{v}] d s
$$

The finite element spaces are denoted by

$$
\begin{aligned}
& \boldsymbol{U}_{h}=\left\{\boldsymbol{u} \in H(\operatorname{div} ; \Omega):\left.\boldsymbol{u}\right|_{K} \in \boldsymbol{U}(K), K \in \mathcal{T}_{h} ; \boldsymbol{u} \cdot \boldsymbol{n}=0 \text { on } \partial \Omega\right\}, \\
& \boldsymbol{V}_{h}=\left\{\boldsymbol{v} \in H(\operatorname{div} ; \Omega):\left.\boldsymbol{v}\right|_{K} \in \boldsymbol{V}(K), K \in \mathcal{T}_{h} ; \boldsymbol{v} \cdot \boldsymbol{n}=0 \text { on } \partial \Omega\right\}, \\
& P_{h}=\left\{q \in L^{2}(\Omega):\left.q\right|_{K} \in Q(K), K \in \mathcal{T}_{h} ; \int_{\Omega} q d x=0\right\} .
\end{aligned}
$$

The discretizations that we consider here define the local spaces $\boldsymbol{U}(K) / \boldsymbol{V}(K) / Q(K)$ via the triplets $B D M_{l}(K) / R T_{l-1}(K) / P_{l-1}(K)$, or $B D F M_{l}(K) / R T_{l-1}(K) / P_{l-1}(K)$ for $l \geq 1$. Note that for all these choices the important condition $\operatorname{div} \boldsymbol{U}(K)=\operatorname{div} \boldsymbol{V}(K)=Q(K)$ is satisfied.

We recall the basic approximation properties of these spaces: for all $K \in \mathcal{T}_{h}$ and for all $\boldsymbol{u} \in H^{s}(K)^{d}$, there exists $\boldsymbol{u}_{I} \in \boldsymbol{U}(K)$ such that

$$
\left\|\boldsymbol{u}-\boldsymbol{u}_{I}\right\|_{0, K}+h_{K}\left|\boldsymbol{u}-\boldsymbol{u}_{I}\right|_{1, K}+h_{K}^{2}\left|\boldsymbol{u}-\boldsymbol{u}_{I}\right|_{2, K} \leq C h_{K}^{s}|\boldsymbol{u}|_{s, K}, \quad 2 \leq s \leq l+1 .
$$

4.2. DG discretization. We note that according to the definition of $\boldsymbol{U}_{h}$, the normal component of any $\boldsymbol{u} \in \boldsymbol{U}_{h}$ is continuous on the internal edges and vanishes on the boundary edges. Therefore, by splitting a vector $\boldsymbol{u} \in \boldsymbol{U}_{h}$ into its normal and tangential components $\boldsymbol{u}_{n}:=(\boldsymbol{u} \cdot \boldsymbol{n}) \boldsymbol{n}$ and $\boldsymbol{u}_{t}:=\boldsymbol{u}-\boldsymbol{u}_{n}$, we have

$$
\text { for all } e \in \mathcal{E}_{h}: \int_{e}\left[\boldsymbol{u}_{n}\right] \cdot \boldsymbol{\tau} d s=0, \quad \text { for all } \boldsymbol{\tau} \in H^{1}\left(\mathcal{T}_{h}\right)^{d}, \boldsymbol{u} \in \boldsymbol{U}_{h},
$$

implying that

$$
\text { for all } e \in \mathcal{E}_{h}: \int_{e}[\boldsymbol{u}] \cdot \boldsymbol{\tau} d s=\int_{e}\left[\boldsymbol{u}_{t}\right] \cdot \boldsymbol{\tau} d s, \quad \text { for all } \boldsymbol{\tau} \in H^{1}\left(\mathcal{T}_{h}\right)^{d}, \boldsymbol{u} \in \boldsymbol{U}_{h} .
$$

A direct computation shows that

$$
\llbracket \boldsymbol{u}_{t} \rrbracket: \llbracket \boldsymbol{w}_{t} \rrbracket=\frac{1}{2}\left[\boldsymbol{u}_{t}\right] \cdot\left[\boldsymbol{w}_{t}\right] .
$$

Therefore, the discretization of the variational problem in equation (2.4) is given as follows: find $\left(\boldsymbol{u}_{h}, \boldsymbol{v}_{h}, p_{h}\right) \in \boldsymbol{U}_{h} \times \boldsymbol{V}_{h} \times P_{h}$ such that for any $\left(\boldsymbol{w}_{h}, \boldsymbol{z}_{h}, q_{h}\right) \in \boldsymbol{U}_{h} \times \boldsymbol{V}_{h} \times P_{h}$

$$
\begin{aligned}
a_{h}\left(\boldsymbol{u}_{h}, \boldsymbol{w}_{h}\right)+\lambda\left(\operatorname{div} \boldsymbol{u}_{h}, \operatorname{div} \boldsymbol{w}_{h}\right)-\left(p_{h}, \operatorname{div} \boldsymbol{w}_{h}\right) & =\left(\boldsymbol{f}, \boldsymbol{w}_{h}\right), \\
R_{p}^{-1}\left(\boldsymbol{v}_{h}, \boldsymbol{z}_{h}\right)-\left(p_{h}, \operatorname{div} \boldsymbol{z}_{h}\right) & =0, \\
-\left(\operatorname{div} \boldsymbol{u}_{h}, q_{h}\right)-\left(\operatorname{div} \boldsymbol{v}_{h}, q_{h}\right)-\alpha_{p}\left(p_{h}, q_{h}\right) & =\left(g, q_{h}\right),
\end{aligned}
$$


where

$$
\begin{aligned}
a_{h}(\boldsymbol{u}, \boldsymbol{w})= & \sum_{K \in \mathcal{T}_{h}} \int_{K} \varepsilon(\boldsymbol{u}): \varepsilon(\boldsymbol{w}) d x-\sum_{e \in \mathcal{E}_{h}} \int_{e}\{\varepsilon(\boldsymbol{u})\} \cdot\left[\boldsymbol{w}_{t}\right] d s \\
& -\sum_{e \in \mathcal{E}_{h}} \int_{e}\{\varepsilon(\boldsymbol{w})\} \cdot\left[\boldsymbol{u}_{t}\right] d s+\sum_{e \in \mathcal{E}_{h}} \int_{e} \eta h_{e}^{-1}\left[\boldsymbol{u}_{t}\right] \cdot\left[\boldsymbol{w}_{t}\right] d s
\end{aligned}
$$

and $\eta$ is a stabilization parameter which is independent of $h, \lambda, R_{p}^{-1}, \alpha_{p}$.

REMARK 4.1. Consider the general rescaled boundary conditions

$$
\begin{aligned}
p & =p_{D} & & \text { on } \Gamma_{p, D}, \\
\boldsymbol{v} \cdot \boldsymbol{n} & =q_{N} & & \text { on } \Gamma_{p, N}, \\
\boldsymbol{u} & =\boldsymbol{u}_{D} & & \text { on } \Gamma_{\boldsymbol{u}, D} \\
(\boldsymbol{\sigma}-p \boldsymbol{I}) \boldsymbol{n} & =\boldsymbol{g}_{N} & & \text { on } \Gamma_{\boldsymbol{u}, N} .
\end{aligned}
$$

Usually, it is assumed that the measure of $\Gamma_{\boldsymbol{u}, D}$ is nonzero to guarantee the discrete Korn's inequality [10]. The standard way to incorporate the boundary conditions (4.3) is to modify the trial spaces according to the boundary conditions, i.e., to seek the solution in the spaces

$$
\begin{aligned}
& \boldsymbol{U}_{h}^{D}=\left\{\boldsymbol{u} \in H(\operatorname{div} ; \Omega):\left.\boldsymbol{u}\right|_{K} \in \boldsymbol{U}(K), K \in \mathcal{T}_{h} ; \boldsymbol{u} \cdot \boldsymbol{n}=\boldsymbol{u}_{D} \cdot \boldsymbol{n} \text { on } \Gamma_{\boldsymbol{u}, D}\right\}, \\
& \boldsymbol{V}_{h}^{D}=\left\{\boldsymbol{v} \in H(\operatorname{div} ; \Omega):\left.\boldsymbol{v}\right|_{K} \in \boldsymbol{V}(K), K \in \mathcal{T}_{h} ; \boldsymbol{v} \cdot \boldsymbol{n}=q_{N} \text { on } \Gamma_{p, N}\right\}, \\
& P_{h}=\left\{\begin{array}{l}
\left\{q \in L^{2}(\Omega):\left.q\right|_{K} \in Q(K), K \in \mathcal{T}_{h}, \text { if }\left|\Gamma_{p, D}\right| \neq 0\right\}, \\
\left\{q \in L_{0}^{2}(\Omega):\left.q\right|_{K} \in Q(K), K \in \mathcal{T}_{h}, \text { if } \Gamma_{p, N}=\Gamma\right\},
\end{array}\right.
\end{aligned}
$$

and use the test spaces given by

$$
\begin{aligned}
& \boldsymbol{U}_{h}^{0}=\left\{\boldsymbol{u} \in H(\operatorname{div} ; \Omega):\left.\boldsymbol{u}\right|_{K} \in \boldsymbol{U}(K), K \in \mathcal{T}_{h} ; \boldsymbol{u} \cdot \boldsymbol{n}=0 \text { on } \Gamma_{\boldsymbol{u}, D}\right\}, \\
& \boldsymbol{V}_{h}^{0}=\left\{\boldsymbol{v} \in H(\operatorname{div} ; \Omega):\left.\boldsymbol{v}\right|_{K} \in \boldsymbol{V}(K), K \in \mathcal{T}_{h} ; \boldsymbol{v} \cdot \boldsymbol{n}=0 \text { on } \Gamma_{p, N}\right\} .
\end{aligned}
$$

Hence, problem (4.1) has the more general formulation: find $\left(\boldsymbol{u}_{h}, \boldsymbol{v}_{h}, p_{h}\right) \in \boldsymbol{U}_{h}^{D} \times \boldsymbol{V}_{h}^{D} \times P_{h}$ such that for any $\left(\boldsymbol{w}_{h}, \boldsymbol{z}_{h}, q_{h}\right) \in \boldsymbol{U}_{h}^{0} \times \boldsymbol{V}_{h}^{0} \times P_{h}$

$$
\begin{aligned}
a_{h}\left(\boldsymbol{u}_{h}, \boldsymbol{w}_{h}\right)+\lambda\left(\operatorname{div} \boldsymbol{u}_{h}, \operatorname{div} \boldsymbol{w}_{h}\right)-\left(p_{h}, \operatorname{div} \boldsymbol{w}_{h}\right) & =F\left(\boldsymbol{w}_{h}\right), \\
\left(R_{p}^{-1} \boldsymbol{v}_{h}, \boldsymbol{z}_{h}\right)-\left(p_{h}, \operatorname{div} \boldsymbol{z}_{h}\right) & =-\left(p_{D}, \boldsymbol{z}_{h} \cdot \boldsymbol{n}\right)_{\Gamma_{p, D}}, \\
-\left(\operatorname{div} \boldsymbol{u}_{h}, q_{h}\right)-\left(\operatorname{div} \boldsymbol{v}_{h}, q_{h}\right)-\alpha_{p}\left(p_{h}, q_{h}\right) & =\left(g, q_{h}\right),
\end{aligned}
$$

where

$$
\begin{aligned}
& a_{h}(\boldsymbol{u}, \boldsymbol{w})= \sum_{K \in \mathcal{T}_{h}} \int_{K} \varepsilon(\boldsymbol{u}): \varepsilon(\boldsymbol{w}) d x-\sum_{e \in \mathcal{E}_{h}^{I} \cup \mathcal{E}_{h}^{\boldsymbol{u}, D}} \int_{e}\{\varepsilon(\boldsymbol{u})\} \cdot\left[\boldsymbol{w}_{t}\right] d s \\
&-\sum_{e \in \mathcal{E}_{h}^{I} \cup \mathcal{E}_{h}^{\boldsymbol{u}, D}} \int_{e}\{\varepsilon(\boldsymbol{w})\} \cdot\left[\boldsymbol{u}_{t}\right] d s+\sum_{e \in \mathcal{E}_{h}^{I} \cup \mathcal{E}_{h}^{u, D}} \int_{e} \eta h_{e}^{-1}\left[\boldsymbol{u}_{t}\right] \cdot\left[\boldsymbol{w}_{t}\right] d s \\
& F(\boldsymbol{w})=(\boldsymbol{f}, \boldsymbol{w})+\left(\boldsymbol{g}_{N}, \boldsymbol{w}\right)_{\Gamma \boldsymbol{u}, N}-\left(\boldsymbol{u}_{D, t}, \varepsilon(\boldsymbol{w}) \boldsymbol{n}\right)_{\Gamma_{\boldsymbol{u}, D}}+\sum_{e \in \mathcal{E}_{h}^{\boldsymbol{u}, D}} \int_{e} \eta h_{e}^{-1} \boldsymbol{u}_{D, t} \cdot \boldsymbol{w}_{t} d s,
\end{aligned}
$$

and $\boldsymbol{u}_{D, t}=\boldsymbol{u}_{D}-\left(\boldsymbol{u}_{D} \cdot \boldsymbol{n}\right) \boldsymbol{n}, \mathcal{E}_{h}^{\boldsymbol{u}, D}=\mathcal{E}_{h}^{B} \cap \Gamma_{\boldsymbol{u}, D}$, and $\eta$ is again a stabilization parameter which is independent of $h, \lambda, R_{p}^{-1}$, and $\alpha_{p}$. If $\Gamma_{\boldsymbol{u}, D}=\Gamma_{p, N}=\Gamma$ and $\boldsymbol{u}_{D}=0, q_{N}=0$, then 
(4.4) reduces to (4.1) which will be analyzed in the remainder of this paper. If the measure of $\Gamma_{\boldsymbol{u}, N}$ is nonzero, then the analysis is similar. If $\Gamma_{\boldsymbol{u}, D}=\Gamma$ and the measure of $\Gamma_{p, D}$ is nonzero, then one has to introduce the modified norms according to (3.3). This part of the analysis is left as future work.

PROPOSITION 4.2. Let $\left(\boldsymbol{u}_{h}, \boldsymbol{v}_{h}, p_{h}\right) \in \boldsymbol{U}_{h} \times \boldsymbol{V}_{h} \times P_{h}$ be the solution of (4.1), then $\left(\boldsymbol{u}_{h}, \boldsymbol{v}_{h}, p_{h}\right)$ satisfy the pointwise mass conservation equation

$$
-\operatorname{div} \boldsymbol{u}_{h}-\operatorname{div} \boldsymbol{v}_{h}-\alpha_{p} p_{h}=Q_{h} g, \quad \forall x \in K, \forall K \in \mathcal{T}_{h},
$$

where $Q_{h}$ denotes the $L^{2}$-projection on $P_{h}$. Further, if $g=0$, then

$$
-\operatorname{div} \boldsymbol{u}_{h}-\operatorname{div} \boldsymbol{v}_{h}-\alpha_{p} p_{h}=0 .
$$

Proof. Since the choice of the spaces satisfying $\nabla \cdot \boldsymbol{U}_{h}=P_{h}$ and $\nabla \cdot \boldsymbol{V}_{h}=P_{h}$ implies

$$
-\operatorname{div} \boldsymbol{u}_{h}-\operatorname{div} \boldsymbol{v}_{h}-\alpha_{p} p_{h} \in P_{h},
$$

equation (4.5) follows directly from the last equation of (4.1).

For any $\boldsymbol{u} \in \boldsymbol{U}_{h}$, we introduce the mesh dependent norms:

$$
\begin{gathered}
\|\boldsymbol{u}\|_{h}^{2}=\sum_{K \in \mathcal{T}_{h}}\|\varepsilon(\boldsymbol{u})\|_{0, K}^{2}+\sum_{e \in \mathcal{E}_{h}} h_{e}^{-1}\left\|\left[\boldsymbol{u}_{t}\right]\right\|_{0, e}^{2}, \\
\|\boldsymbol{u}\|_{1, h}^{2}=\sum_{K \in \mathcal{T}_{h}}\|\nabla \boldsymbol{u}\|_{0, K}^{2}+\sum_{e \in \mathcal{E}_{h}} h_{e}^{-1}\left\|\left[\boldsymbol{u}_{t}\right]\right\|_{0, e}^{2} .
\end{gathered}
$$

Next, for $\boldsymbol{u} \in \boldsymbol{U}_{h}$, we define the "DG"-norm

$$
\|\boldsymbol{u}\|_{D G}^{2}=\sum_{K \in \mathcal{T}_{h}}\|\nabla \boldsymbol{u}\|_{0, K}^{2}+\sum_{e \in \mathcal{E}_{h}} h_{e}^{-1}\left\|\left[\boldsymbol{u}_{t}\right]\right\|_{0, e}^{2}+\sum_{K \in \mathcal{T}_{h}} h_{K}^{2}|\boldsymbol{u}|_{2, K}^{2}
$$

and, finally, the mesh-dependent norm $\|\cdot\|_{\boldsymbol{U}_{h}}$ by

$$
\|\boldsymbol{u}\|_{\boldsymbol{U}_{h}}^{2}=\|\boldsymbol{u}\|_{D G}^{2}+\lambda\|\operatorname{div} \boldsymbol{u}\|^{2} .
$$

We now summarize several results on well-posedness and approximation properties of the DG formulation; see, e.g., [23, 22].

- From the discrete version of Korn's inequality we have that the norms $\|\cdot\|_{D G},\|\cdot\|_{h}$, and $\|\cdot\|_{1, h}$ are equivalent on $\boldsymbol{U}_{h}$, namely,

$$
\|\boldsymbol{u}\|_{D G} \approx\|\boldsymbol{u}\|_{h} \approx\|\boldsymbol{u}\|_{1, h}, \quad \text { for all } \boldsymbol{u} \in \boldsymbol{U}_{h} .
$$

- The bilinear form $a_{h}(\cdot, \cdot)$, introduced in (4.2) is continuous and we have

$$
\left|a_{h}(\boldsymbol{u}, \boldsymbol{w})\right| \lesssim\|\boldsymbol{u}\|_{D G}\|\boldsymbol{w}\|_{D G}, \quad \text { for all } \boldsymbol{u}, \boldsymbol{w} \in H^{2}\left(\mathcal{T}_{h}\right)^{d} .
$$

- For our choice of the finite element spaces $\boldsymbol{V}_{h}$ and $P_{h}$ we have the following inf-sup conditions; see, e.g., [34].

$$
\begin{gathered}
\inf _{q_{h} \in P_{h}} \sup _{\boldsymbol{u}_{h} \in \boldsymbol{U}_{h}} \frac{\left(\operatorname{div} \boldsymbol{u}_{h}, q_{h}\right)}{\left\|\boldsymbol{u}_{h}\right\|_{1, h}\left\|q_{h}\right\|} \geq \beta_{s d}>0, \\
\inf _{q_{h} \in P_{h}} \sup _{\boldsymbol{v}_{h} \in \boldsymbol{V}_{h}} \frac{\left(\operatorname{div} \boldsymbol{v}_{h}, q_{h}\right)}{\left\|\boldsymbol{v}_{h}\right\|_{\operatorname{div}}\left\|q_{h}\right\|} \geq \beta_{d d}>0,
\end{gathered}
$$

where $\beta_{s d}$ and $\beta_{d d}$ are constants independent of the parameters $\lambda, R_{p}^{-1}, \alpha_{p}$ and the mesh size $h$. 
- We also have that $a_{h}(\cdot, \cdot)$ is coercive:

$$
a_{h}\left(\boldsymbol{u}_{h}, \boldsymbol{u}_{h}\right) \geq \alpha_{a}\left\|\boldsymbol{u}_{h}\right\|_{h}^{2}, \quad \text { for all } \boldsymbol{u}_{h} \in \boldsymbol{U}_{h},
$$

where $\alpha_{a}$ is a positive constant independent of the parameters $\lambda, R_{p}^{-1}, \alpha_{p}$, and the mesh size $h$.

Related to the discrete problem (4.1) we introduce the bilinear form

$$
\begin{aligned}
A_{h}\left(\left(\boldsymbol{u}_{h}, \boldsymbol{v}_{h}, p_{h}\right),\left(\boldsymbol{w}_{h}, \boldsymbol{z}_{h}, q_{h}\right)\right)=a_{h} & \left(\boldsymbol{u}_{h}, \boldsymbol{v}_{h}\right)+\lambda\left(\operatorname{div} \boldsymbol{u}_{h}, \operatorname{div} \boldsymbol{w}_{h}\right) \\
& -\left(p_{h}, \operatorname{div} \boldsymbol{w}_{h}\right)+R_{p}^{-1}\left(\boldsymbol{v}_{h}, \boldsymbol{z}_{h}\right)-\left(p_{h}, \operatorname{div} \boldsymbol{z}_{h}\right) \\
& -\left(\operatorname{div} \boldsymbol{u}_{h}, q_{h}\right)-\left(\operatorname{div} \boldsymbol{v}_{h}, q_{h}\right)-\alpha_{p}\left(p_{h}, q_{h}\right) .
\end{aligned}
$$

In view of the definitions of the norms $\|\cdot\|_{U_{h}},\|\cdot\|_{V}$, and $\|\cdot\|_{P}$, the boundedness of $A_{h}\left(\left(\boldsymbol{u}_{h}, \boldsymbol{v}_{h}, p_{h}\right),\left(\boldsymbol{w}_{h}, \boldsymbol{z}_{h}, q_{h}\right)\right)$ is obvious, i.e., the following theorem holds.

THEOREM 4.3. There exists a constant $C_{b d}$ independent of the parameters $\lambda, R_{p}^{-1}, \alpha_{p}$, and the mesh size $h$ such that for any $\left(\boldsymbol{u}_{h}, \boldsymbol{v}_{h}, p_{h}\right) \in \boldsymbol{U}_{h} \times \boldsymbol{V}_{h} \times P_{h}$ and $\left(\boldsymbol{w}_{h}, \boldsymbol{z}_{h}, q_{h}\right) \in$ $\boldsymbol{U}_{h} \times \boldsymbol{V}_{h} \times P_{h}$ it holds that

$$
\begin{aligned}
& \left|A_{h}\left(\left(\boldsymbol{u}_{h}, \boldsymbol{v}_{h}, p_{h}\right),\left(\boldsymbol{w}_{h}, \boldsymbol{z}_{h}, q_{h}\right)\right)\right| \\
& \quad \leq C_{b d}\left(\left\|\boldsymbol{u}_{h}\right\|_{\boldsymbol{U}_{h}}+\left\|\boldsymbol{v}_{h}\right\|_{\boldsymbol{V}}+\left\|p_{h}\right\|_{P}\right)\left(\left\|\boldsymbol{w}_{h}\right\|_{\boldsymbol{U}_{h}}+\left\|\boldsymbol{z}_{h}\right\|_{\boldsymbol{V}}+\left\|q_{h}\right\|_{P}\right) .
\end{aligned}
$$

We come to our second main result.

THEOREM 4.4. There exits a constant $\beta_{0}>0$ independent of the parameters $\lambda, R_{p}^{-1}, \alpha_{p}$, and the mesh size $h$ such that

$$
\inf _{\substack{\left(\boldsymbol{u}_{h}, \boldsymbol{v}_{h}, p_{h}\right) \\ \in \boldsymbol{U}_{h} \times \boldsymbol{V}_{h} \times P_{h}}} \sup _{\substack{\left(\boldsymbol{w}_{h}, \boldsymbol{z}_{h}, q_{h}\right) \\ \in \boldsymbol{U}_{h} \times \boldsymbol{V}_{h} \times P_{h}}} \frac{A_{h}\left(\left(\boldsymbol{u}_{h}, \boldsymbol{v}_{h}, p_{h}\right),\left(\boldsymbol{w}_{h}, \boldsymbol{z}_{h}, q_{h}\right)\right)}{\left(\left\|\boldsymbol{u}_{h}\right\|_{\boldsymbol{U}_{h}}+\left\|\boldsymbol{v}_{h}\right\|_{\boldsymbol{V}}+\left\|p_{h}\right\|_{P}\right)\left(\left\|\boldsymbol{w}_{h}\right\|_{\boldsymbol{U}_{h}}+\left\|\boldsymbol{z}_{h}\right\|_{\boldsymbol{V}}+\left\|q_{h}\right\|_{P}\right)} \geq \beta_{0} .
$$

Proof. Case I:

$$
\rho=\min \left\{\lambda, R_{p}^{-1}\right\}=\lambda, \quad \text { hence } \quad \lambda \leq R_{p}^{-1}, \quad \gamma^{-1} \leq \rho=\lambda .
$$

For any $\left(\boldsymbol{u}_{h}, \boldsymbol{v}_{h}, p_{h}\right) \in \boldsymbol{U}_{h} \times \boldsymbol{V}_{h} \times P_{h}$, by the first inf-sup condition in (4.7), there exists

$$
\boldsymbol{u}_{h, 0} \in \boldsymbol{U}_{h} \text { such that } \quad \operatorname{div} \boldsymbol{u}_{h, 0}=\frac{1}{\sqrt{\lambda}} p_{h}, \quad\left\|\boldsymbol{u}_{h, 0}\right\|_{1, h} \leq \beta_{s d}^{-1} \frac{1}{\sqrt{\lambda}}\left\|p_{h}\right\| .
$$

We choose

$$
\boldsymbol{w}_{h}=\delta \boldsymbol{u}_{h}-\frac{1}{\sqrt{\lambda}} \boldsymbol{u}_{h, 0}, \boldsymbol{z}_{h}=\delta \boldsymbol{v}_{h}, \quad q_{h}=-\delta p_{h}-\gamma^{-1} \operatorname{div} \boldsymbol{v}_{h},
$$

where the constant $\delta$ will be determined later.

We verify first the boundedness of $\left(\boldsymbol{w}_{h}, \boldsymbol{z}_{h}, q_{h}\right)$ by $\left(\boldsymbol{u}_{h}, \boldsymbol{v}_{h}, p_{h}\right)$. By (4.11), the equivalence between the norms $\|\cdot\|_{D G}$ and $\|\cdot\|_{1, h}$, namely (4.6), and noting that $\gamma^{-1} \leq \rho=\lambda$, we have

$$
\begin{aligned}
&\left\|\frac{1}{\sqrt{\lambda}} \boldsymbol{u}_{h, 0}\right\|_{\boldsymbol{U}_{h}}^{2}=\left\|\frac{1}{\sqrt{\lambda}} \boldsymbol{u}_{h, 0}\right\|_{D G}^{2}+\lambda\left(\operatorname{div} \frac{1}{\sqrt{\lambda}} \boldsymbol{u}_{h, 0}, \operatorname{div} \frac{1}{\sqrt{\lambda}} \boldsymbol{u}_{h, 0}\right) \\
& \leq \text { by }(4.6) \\
& C_{0}^{2}\left\|\frac{1}{\sqrt{\lambda}} \boldsymbol{u}_{h, 0}\right\|_{1, h}^{2}+\left(\operatorname{div} \boldsymbol{u}_{h, 0}, \operatorname{div} \boldsymbol{u}_{h, 0}\right) \\
& \leq \frac{1}{\lambda} C_{0}^{2} \beta_{s d}^{-2} \frac{1}{\lambda}\|p\|^{2}+\frac{1}{\lambda}(p, p) \leq\left(\frac{1}{\lambda} C_{0}^{2} \beta_{s d}^{-2}+1\right) \frac{1}{\lambda}\left\|p_{h}\right\|^{2} \\
& \leq\left(\frac{1}{\lambda} C_{0}^{2} \beta_{s d}^{-2}+1\right) \gamma\left\|p_{h}\right\|^{2}=\left(\frac{1}{\lambda} C_{0}^{2} \beta_{s d}^{-2}+1\right)\left(p_{h}, p_{h}\right)_{P} .
\end{aligned}
$$


Therefore, by taking into account that $1 \leq \lambda$ we get for $\boldsymbol{w}_{h}$ the estimate

$$
\left\|\boldsymbol{w}_{h}\right\|_{\boldsymbol{U}_{h}} \leq \delta\left\|\boldsymbol{u}_{h}\right\|_{\boldsymbol{U}_{h}}+\sqrt{\lambda^{-1} C_{0}^{2} \beta_{s d}^{-2}+1}\left\|p_{h}\right\|_{P} \leq \delta\left\|\boldsymbol{u}_{h}\right\|_{\boldsymbol{U}_{h}}+\sqrt{C_{0}^{2} \beta_{s d}^{-2}+1}\left\|p_{h}\right\|_{P} .
$$

Obviously we have $\left\|\boldsymbol{z}_{h}\right\|_{\boldsymbol{V}}=\delta\left\|\boldsymbol{v}_{h}\right\|_{\boldsymbol{V}}$ and it remains to bound $q_{h}$. From the fact that

$$
\begin{aligned}
\left(\gamma^{-1} \operatorname{div} \boldsymbol{v}_{h}, \gamma^{-1} \operatorname{div} \boldsymbol{v}_{h}\right)_{P} & =\gamma\left(\gamma^{-1} \operatorname{div} \boldsymbol{v}_{h}, \gamma^{-1} \operatorname{div} \boldsymbol{v}_{h}\right) \\
& =\gamma^{-1}\left(\operatorname{div} \boldsymbol{v}_{h}, \operatorname{div} \boldsymbol{v}_{h}\right) \leq\left(\boldsymbol{v}_{h}, \boldsymbol{v}_{h}\right)_{\boldsymbol{V}}
\end{aligned}
$$

it follows that

$$
\left\|q_{h}\right\|_{P} \leq \delta\left\|p_{h}\right\|_{P}+\left\|\boldsymbol{v}_{h}\right\|_{\boldsymbol{V}}
$$

Using the definition of $\left(\boldsymbol{w}_{h}, \boldsymbol{z}_{h}, q_{h}\right)$ and equation (4.11), we establish the coercivity of $A_{h}\left(\left(\boldsymbol{u}_{h}, \boldsymbol{v}_{h}, p_{h}\right),\left(\boldsymbol{w}_{h}, \boldsymbol{z}_{h}, q_{h}\right)\right)$ :

$$
\begin{aligned}
A_{h}\left(\left(\boldsymbol{u}_{h}, \boldsymbol{v}_{h}, p_{h}\right),\left(\boldsymbol{w}_{h}, \boldsymbol{z}_{h}, q_{h}\right)\right) \\
=a_{h}\left(\boldsymbol{u}_{h}, \boldsymbol{w}_{h}\right)+\lambda\left(\operatorname{div} \boldsymbol{u}_{h}, \operatorname{div} \boldsymbol{w}_{h}\right)-\left(p_{h}, \operatorname{div} \boldsymbol{w}_{h}\right)+R_{p}^{-1}\left(\boldsymbol{v}_{h}, \boldsymbol{z}_{h}\right)-\left(p_{h}, \operatorname{div} \boldsymbol{z}_{h}\right) \\
\quad-\left(\operatorname{div} \boldsymbol{u}_{h}, q_{h}\right)-\left(\operatorname{div} \boldsymbol{v}_{h}, q_{h}\right)-\alpha_{p}\left(p_{h}, q_{h}\right) \\
=a_{h}\left(\boldsymbol{u}_{h}, \delta \boldsymbol{u}_{h}-\frac{1}{\sqrt{\lambda}} \boldsymbol{u}_{h, 0}\right)+\lambda\left(\operatorname{div} \boldsymbol{u}_{h}, \delta \operatorname{div} \boldsymbol{u}_{h}-\frac{1}{\sqrt{\lambda}} \operatorname{div} \boldsymbol{u}_{h, 0}\right) \\
\quad-\left(p_{h}, \delta \operatorname{div} \boldsymbol{u}_{h}-\frac{1}{\sqrt{\lambda}} \operatorname{div} \boldsymbol{u}_{h, 0}\right)+R_{p}^{-1}\left(\boldsymbol{v}_{h}, \delta \boldsymbol{v}_{h}\right)-\left(p_{h}, \delta \operatorname{div} \boldsymbol{v}_{h}\right) \\
\quad-\left(\operatorname{div} \boldsymbol{u}_{h},-\delta p_{h}-\gamma^{-1} \operatorname{div} \boldsymbol{v}_{h}\right)-\left(\operatorname{div} \boldsymbol{v}_{h},-\delta p_{h}-\gamma^{-1} \operatorname{div} \boldsymbol{v}_{h}\right) \\
\quad-\alpha_{p}\left(p_{h},-\delta p_{h}-\gamma^{-1} \operatorname{div} \boldsymbol{v}_{h}\right) \\
=\delta a_{h}\left(\boldsymbol{u}_{h}, \boldsymbol{u}_{h}\right)-\frac{1}{\sqrt{\lambda}} a_{h}\left(\boldsymbol{u}_{h}, \boldsymbol{u}_{h, 0}\right)+\delta \lambda\left(\operatorname{div} \boldsymbol{u}_{h}, \operatorname{div} \boldsymbol{u}_{h}\right)-\sqrt{\lambda}\left(\operatorname{div} \boldsymbol{u}_{h}, \operatorname{div} \boldsymbol{u}_{h, 0}\right) \\
\quad-\delta\left(p_{h}, \operatorname{div} \boldsymbol{u}_{h}\right)+\frac{1}{\sqrt{\lambda}}\left(p_{h}, \operatorname{div} \boldsymbol{u}_{h, 0}\right)+\delta R_{p}^{-1}\left(\boldsymbol{v}_{h}, \boldsymbol{v}_{h}\right) \\
\quad-\delta\left(p_{h}, \operatorname{div} \boldsymbol{v}_{h}\right)+\delta\left(\operatorname{div} \boldsymbol{u}_{h}, p_{h}\right)+\gamma^{-1}\left(\operatorname{div} \boldsymbol{u}_{h}, \operatorname{div} \boldsymbol{v}_{h}\right)+\delta\left(\operatorname{div} \boldsymbol{v}_{h}, p_{h}\right) \\
\quad+\gamma^{-1}\left(\operatorname{div} \boldsymbol{v}_{h}, \operatorname{div} \boldsymbol{v}_{h}\right)+\delta \alpha_{p}\left(p_{h}, p_{h}\right)+\alpha_{p}\left(p_{h}, \gamma^{-1} \operatorname{div} \boldsymbol{v}_{h}\right) \\
={ }_{\operatorname{by}(4.11)} \delta a_{h}\left(\boldsymbol{u}_{h}, \boldsymbol{u}_{h}\right)-\frac{1}{\sqrt{\lambda}} a_{h}\left(\boldsymbol{u}_{h}, \boldsymbol{u}_{h, 0}\right)+\delta \lambda\left(\operatorname{div} \boldsymbol{u}_{h}, \operatorname{div} \boldsymbol{u}_{h}\right)-\left(\operatorname{div} \boldsymbol{u}_{h}, p_{h}\right) \\
\quad+\frac{1}{\lambda}\left(p_{h}, p_{h}\right)+\delta R_{p}^{-1}\left(\boldsymbol{v}_{h}, \boldsymbol{v}_{h}\right)+\gamma^{-1}\left(\operatorname{div} \boldsymbol{u}_{h}, \operatorname{div} \boldsymbol{v}_{h}\right)+\gamma^{-1}\left(\operatorname{div} \boldsymbol{v}_{h}, \operatorname{div} \boldsymbol{v}_{h}\right) \\
\quad+\delta \alpha_{p}\left(p_{h}, p_{h}\right)+\alpha_{p}\left(p_{h}, \gamma^{-1} \operatorname{div} \boldsymbol{v}_{h}\right) .
\end{aligned}
$$

Next we apply Young's inequality, use the coercivity and the continuity of $a_{h}(\cdot, \cdot)$, the 
equivalence of the norms $\|\cdot\|_{D G}$ and $\|\cdot\|_{1, h}$, and (4.11) to get

$$
\begin{aligned}
A_{h}\left(\left(\boldsymbol{u}_{h}, \boldsymbol{v}_{h}, p_{h}\right),\left(\boldsymbol{w}_{h}, \boldsymbol{z}_{h}, p_{h}\right)\right) \\
=\delta a_{h}\left(\boldsymbol{u}_{h}, \boldsymbol{u}_{h}\right)-\frac{1}{\sqrt{\lambda}} a_{h}\left(\boldsymbol{u}_{h}, \boldsymbol{u}_{h, 0}\right)+\delta \lambda\left(\operatorname{div} \boldsymbol{u}_{h}, \operatorname{div} \boldsymbol{u}_{h}\right)-\left(\operatorname{div} \boldsymbol{u}_{h}, p_{h}\right)+\frac{1}{\lambda}\left(p_{h}, p_{h}\right) \\
\quad+\delta R_{p}^{-1}\left(\boldsymbol{v}_{h}, \boldsymbol{v}_{h}\right)+\gamma^{-1}\left(\operatorname{div} \boldsymbol{u}_{h}, \operatorname{div} \boldsymbol{v}_{h}\right)+\gamma^{-1}\left(\operatorname{div} \boldsymbol{v}_{h}, \operatorname{div} \boldsymbol{v}_{h}\right) \\
\quad+\delta \alpha_{p}\left(p_{h}, p_{h}\right)+\alpha_{p}\left(p_{h}, \gamma^{-1} \operatorname{div} \boldsymbol{v}_{h}\right) \\
\geq \delta a_{h}\left(\boldsymbol{u}_{h}, \boldsymbol{u}_{h}\right)-\frac{1}{2} \frac{1}{\sqrt{\lambda}} \epsilon_{1}^{-1} a_{h}\left(\boldsymbol{u}_{h}, \boldsymbol{u}_{h}\right)-\frac{1}{2} \frac{1}{\sqrt{\lambda}} \epsilon_{1} a_{h}\left(\boldsymbol{u}_{h, 0}, \boldsymbol{u}_{h, 0}\right)+\delta \lambda\left(\operatorname{div} \boldsymbol{u}_{h}, \operatorname{div} \boldsymbol{u}_{h}\right) \\
\quad-\frac{1}{2} \epsilon_{2}^{-1} \lambda\left(\operatorname{div} \boldsymbol{u}_{h}, \operatorname{div} \boldsymbol{u}_{h}\right)-\frac{1}{2} \epsilon_{2} \frac{1}{\lambda}\left(p_{h}, p_{h}\right)+\frac{1}{\lambda}\left(p_{h}, p_{h}\right)+\delta R_{p}^{-1}\left(\boldsymbol{v}_{h}, \boldsymbol{v}_{h}\right) \\
\quad-\frac{1}{2} \epsilon_{3}^{-1} \gamma^{-1}\left(\operatorname{div} \boldsymbol{u}_{h}, \operatorname{div} \boldsymbol{u}_{h}\right)-\frac{1}{2} \epsilon_{3} \gamma^{-1}\left(\operatorname{div} \boldsymbol{v}_{h}, \operatorname{div} \boldsymbol{v}_{h}\right)+\gamma^{-1}\left(\operatorname{div} \boldsymbol{v}_{h}, \operatorname{div} \boldsymbol{v}_{h}\right) \\
\quad+\delta \alpha_{p}\left(p_{h}, p_{h}\right)-\frac{1}{2} \epsilon_{4} \gamma^{-1}\left(\operatorname{div} \boldsymbol{v}_{h}, \operatorname{div} \boldsymbol{v}_{h}\right)-\frac{1}{2} \epsilon_{4}^{-1} \alpha_{p}^{2} \gamma^{-1}\left(p_{h}, p_{h}\right) \\
\geq \operatorname{by}(4.11),(4.8),(4.6)\left(\delta-\frac{1}{2} \frac{1}{\sqrt{\lambda}} \epsilon_{1}^{-1}\right) \alpha_{a}\left\|\boldsymbol{u}_{h}\right\|_{D G}^{2}+\left(\delta-\frac{1}{2} \epsilon_{2}^{-1}\right) \lambda\left(\operatorname{div} \boldsymbol{u}_{h}, \operatorname{div} \boldsymbol{u}_{h}\right) \\
\quad-\frac{1}{2} \epsilon_{3}^{-1} \gamma^{-1}\left(\operatorname{div} \boldsymbol{u}_{h}, \operatorname{div} \boldsymbol{u}_{h}\right)+\delta R_{p}^{-1}\left(\boldsymbol{v}_{h}, \boldsymbol{v}_{h}\right) \\
\quad+\left(1-\frac{1}{2} \epsilon_{3}-\frac{1}{2} \epsilon_{4}\right) \gamma^{-1}\left(\operatorname{div} \boldsymbol{v}_{h}, \operatorname{div} \boldsymbol{v}_{h}\right)+\left(1-\frac{1}{2} \frac{1}{\sqrt{\lambda}} \epsilon_{1} C_{1}^{2} C_{0}^{2} \beta_{s d}^{-2}-\frac{1}{2} \epsilon_{2}\right) \frac{1}{\lambda}\left(p_{h}, p_{h}\right) \\
\quad+\left(\delta-\frac{1}{2} \epsilon_{4}^{-1} \alpha_{p} \gamma^{-1}\right) \alpha_{p}\left(p_{h}, p_{h}\right) .
\end{aligned}
$$

Now letting $\epsilon_{1}=\frac{1}{2} C_{1}^{-2} C_{0}^{-2} \beta_{s d}^{2}, \epsilon_{2}=\epsilon_{3}=\epsilon_{4}=\frac{1}{2}$ and noting that $\rho^{-1} \leq \gamma, \lambda \geq \gamma^{-1}>0$, we obtain

$$
\begin{aligned}
& A\left(\left(\boldsymbol{u}_{h}, \boldsymbol{v}_{h}, p_{h}\right),\left(\boldsymbol{w}_{h}, \boldsymbol{z}_{h}, p_{h}\right)\right) \\
& \quad \geq\left(\delta-\frac{1}{\sqrt{\lambda}} C_{1}^{2} C_{0}^{2} \beta_{s d}^{-2}\right) \alpha_{a}\left\|\boldsymbol{u}_{h}\right\|_{D G}^{2}+(\delta-2) \lambda\left(\operatorname{div} \boldsymbol{u}_{h}, \operatorname{div} \boldsymbol{u}_{h}\right)+\delta R_{p}^{-1}\left(\boldsymbol{v}_{h}, \boldsymbol{v}_{h}\right) \\
& \quad+\frac{1}{2} \gamma^{-1}\left(\operatorname{div} \boldsymbol{v}_{h}, \operatorname{div} \boldsymbol{v}_{h}\right)+\frac{1}{2} \frac{1}{\lambda}\left(p_{h}, p_{h}\right)+\left(\delta-\alpha_{p} \gamma^{-1}\right) \alpha_{p}\left(p_{h}, p_{h}\right) .
\end{aligned}
$$

Next, setting $\delta:=\max \left\{C_{1}^{2} C_{0}^{2} \beta_{s d}^{-2}+\frac{1}{2} \alpha_{a}^{-1}, 2+\frac{1}{2}\right\}$ and noting that $\alpha_{p} \leq \gamma, \lambda \geq 1$, we derive the coercivity estimate

$$
\begin{aligned}
& A\left(\left(\boldsymbol{u}_{h}, \boldsymbol{v}_{h}, p_{h}\right),\left(\boldsymbol{w}_{h}, \boldsymbol{z}_{h}, p_{h}\right)\right) \\
& \quad \geq \frac{1}{2}\left\|\boldsymbol{u}_{h}\right\|_{D G}^{2}+\frac{1}{2} \lambda\left(\operatorname{div} \boldsymbol{u}_{h}, \operatorname{div} \boldsymbol{u}_{h}\right)+\frac{1}{2} R_{p}^{-1}\left(\boldsymbol{v}_{h}, \boldsymbol{v}_{h}\right) \\
& \quad \quad+\frac{1}{2} \gamma^{-1}\left(\operatorname{div} \boldsymbol{v}_{h}, \operatorname{div} \boldsymbol{v}_{h}\right)+\frac{1}{2} \frac{1}{\lambda}\left(p_{h}, p_{h}\right)+\frac{1}{2} \alpha_{p}\left(p_{h}, p_{h}\right) \\
& \quad \geq \frac{1}{2}\left(\left\|\boldsymbol{u}_{h}\right\|_{\boldsymbol{U}_{h}}^{2}+\left\|\boldsymbol{v}_{h}\right\|_{\boldsymbol{V}}^{2}+\left\|p_{h}\right\|_{P}^{2}\right) .
\end{aligned}
$$

Case II:

$$
\rho=\min \left\{\lambda, R_{p}^{-1}\right\}=R_{p}^{-1}, \quad \text { hence } \quad \lambda \geq R_{p}^{-1}, \quad \gamma^{-1} \leq \rho=R_{p}^{-1} .
$$

For any $\left(\boldsymbol{u}_{h}, \boldsymbol{v}_{h}, p_{h}\right) \in \boldsymbol{U}_{h} \times \boldsymbol{V}_{h} \times P_{h}$, by the second inequality in (4.7), there exists

$$
\boldsymbol{v}_{h, 0} \in \boldsymbol{V}_{h}, \text { such that } \quad \operatorname{div} \boldsymbol{v}_{h, 0}=\sqrt{R_{p}} p_{h}, \quad\left\|\boldsymbol{v}_{h, 0}\right\|_{\operatorname{div}} \leq \beta_{d d}^{-1} \sqrt{R_{p}}\left\|p_{h}\right\| .
$$


We choose

$$
\boldsymbol{w}_{h}=\delta \boldsymbol{u}_{h}, \quad \boldsymbol{z}_{h}=\delta \boldsymbol{v}_{h}-\sqrt{R_{p}} \boldsymbol{v}_{h, 0}, \quad q_{h}=-\delta p_{h}-\gamma^{-1} \operatorname{div} \boldsymbol{v}_{h},
$$

where $\delta$ is a constant which will be specified later.

Again we verify first the boundedness of $\left(\boldsymbol{w}_{h}, \boldsymbol{z}_{h}, q_{h}\right)$ by $\left(\boldsymbol{u}_{h}, \boldsymbol{v}_{h}, p_{h}\right)$. We note that $\left\|\boldsymbol{w}_{h}\right\|_{\boldsymbol{U}_{h}}=\delta\left\|\boldsymbol{u}_{h}\right\|_{\boldsymbol{U}_{h}}$.

From (4.13) and with $\gamma^{-1} \leq \rho=R_{p}^{-1}$ we have

$$
\begin{aligned}
\left(\sqrt{R}_{p} \boldsymbol{v}_{h, 0}, \sqrt{R}_{p} \boldsymbol{v}_{h, 0}\right)_{\boldsymbol{V}} & =R_{p}^{-1}\left(\sqrt{R}_{p} \boldsymbol{v}_{h, 0}, \sqrt{R_{p}} \boldsymbol{v}_{h, 0}\right)+\gamma^{-1}\left(\operatorname{div} \sqrt{R_{p}} \boldsymbol{v}_{h, 0}, \operatorname{div} \sqrt{R_{p}} \boldsymbol{v}_{h, 0}\right) \\
& \leq\left(\boldsymbol{v}_{h, 0}, \boldsymbol{v}_{h, 0}\right)+\left(\operatorname{div} \boldsymbol{v}_{h, 0}, \operatorname{div} \boldsymbol{v}_{h, 0}\right) \\
& \leq \beta_{d d}^{-2} R_{p}\left\|p_{h}\right\|^{2} \leq \beta_{d d}^{-2} \gamma\left\|p_{h}\right\|^{2} \leq \beta_{d d}^{-2}\left(p_{h}, p_{h}\right)_{P} .
\end{aligned}
$$

Hence, we get the boundedness of $\boldsymbol{z}_{h}$, that is,

$$
\left\|\boldsymbol{z}_{h}\right\|_{\boldsymbol{V}} \leq \delta\left\|\boldsymbol{v}_{h}\right\|_{\boldsymbol{V}}+\beta_{d d}^{-1}\left\|p_{h}\right\|_{P}
$$

Again we have the boundedness for $q_{h}$ according to (4.12).

In what follows we show the coercivity of $A_{h}\left(\left(\boldsymbol{u}_{h}, \boldsymbol{v}_{h}, p_{h}\right),\left(\boldsymbol{w}_{h}, \boldsymbol{z}_{h}, q_{h}\right)\right)$ in Case II. Using the definition of $\left(\boldsymbol{w}_{h}, \boldsymbol{z}_{h}, q_{h}\right)$ and (4.13), we find

$$
\begin{aligned}
& A_{h}(\left.\left(\boldsymbol{u}_{h}, \boldsymbol{v}_{h}, p_{h}\right),\left(\boldsymbol{w}_{h}, \boldsymbol{z}_{h}, q_{h}\right)\right) \\
&=a_{h}\left(\boldsymbol{u}_{h}, \boldsymbol{w}_{h}\right)+\lambda\left(\operatorname{div} \boldsymbol{u}_{h}, \operatorname{div} \boldsymbol{w}_{h}\right)-\left(p_{h}, \operatorname{div} \boldsymbol{w}_{h}\right)+R_{p}^{-1}\left(\boldsymbol{v}_{h}, \boldsymbol{z}_{h}\right)-\left(p_{h}, \operatorname{div} \boldsymbol{z}_{h}\right) \quad-\left(\operatorname{div} \boldsymbol{u}_{h}, q_{h}\right)-\left(\operatorname{div} \boldsymbol{v}_{h}, q_{h}\right)-\alpha_{p}\left(p_{h}, q_{h}\right) \\
&=a_{h}\left(\boldsymbol{u}_{h}, \delta \boldsymbol{u}_{h}\right)+\lambda\left(\operatorname{div} \boldsymbol{u}_{h}, \delta \operatorname{div} \boldsymbol{u}_{h}\right)-\left(p_{h}, \delta \operatorname{div} \boldsymbol{u}_{h}\right)+R_{p}^{-1}\left(\boldsymbol{v}_{h}, \delta \boldsymbol{v}_{h}-\sqrt{R_{p}} \boldsymbol{v}_{h, 0}\right) \\
& \quad-\left(p_{h}, \delta \operatorname{div} \boldsymbol{v}_{h}-\sqrt{R_{p}} \operatorname{div} \boldsymbol{v}_{h, 0}\right)-\left(\operatorname{div} \boldsymbol{u}_{h},-\delta p_{h}-\gamma^{-1} \operatorname{div} \boldsymbol{v}_{h}\right) \\
& \quad-\left(\operatorname{div} \boldsymbol{v}_{h},-\delta p_{h}-\gamma^{-1} \operatorname{div} \boldsymbol{v}_{h}\right)-\alpha_{p}\left(p_{h},-\delta p_{h}-\gamma^{-1} \operatorname{div} \boldsymbol{v}_{h}\right) \\
&=\delta a_{h}\left(\boldsymbol{u}_{h}, \boldsymbol{u}_{h}\right)+\delta \lambda\left(\operatorname{div} \boldsymbol{u}_{h}, \operatorname{div} \boldsymbol{u}_{h}\right)-\delta\left(p_{h}, \operatorname{div} \boldsymbol{u}_{h}\right) \\
& \quad+\delta R_{p}^{-1}\left(\boldsymbol{v}_{h}, \boldsymbol{v}_{h}\right)-\left(R_{p}^{-1 / 2} \boldsymbol{v}_{h}, \boldsymbol{v}_{h, 0}\right)-\delta\left(p_{h}, \operatorname{div} \boldsymbol{v}_{h}\right)+\left(p_{h}, \sqrt{R_{p}} \operatorname{div} \boldsymbol{v}_{h, 0}\right) \\
& \quad+\delta\left(\operatorname{div} \boldsymbol{u}_{h}, p_{h}\right)+\gamma^{-1}\left(\operatorname{div} \boldsymbol{u}_{h}, \operatorname{div} \boldsymbol{v}_{h}\right)+\delta\left(\operatorname{div} \boldsymbol{v}_{h}, p_{h}\right)+\gamma^{-1}\left(\operatorname{div} \boldsymbol{v}_{h}, \operatorname{div} \boldsymbol{v}_{h}\right) \\
& \quad+\delta \alpha_{p}\left(p_{h}, p_{h}\right)+\alpha_{p}\left(p_{h}, \gamma^{-1} \operatorname{div} \boldsymbol{v}_{h}\right) \\
&={ }_{\text {by }(4.13)} \delta a_{h}\left(\boldsymbol{u}_{h}, \boldsymbol{u}_{h}\right)+\delta \lambda\left(\operatorname{div} \boldsymbol{u}_{h}, \operatorname{div} \boldsymbol{u}_{h}\right)+\delta R_{p}^{-1}\left(\boldsymbol{v}_{h}, \boldsymbol{v}_{h}\right)-\left(R_{p}^{-1 / 2} \boldsymbol{v}_{h}, \boldsymbol{v}_{h, 0}\right) \\
& \quad+\left(p_{h}, R_{p} p_{h}\right)+\gamma^{-1}\left(\operatorname{div} \boldsymbol{u}_{h}, \operatorname{div} \boldsymbol{v}_{h}\right)+\gamma^{-1}\left(\operatorname{div} \boldsymbol{v}_{h}, \operatorname{div} \boldsymbol{v}_{h}\right) \\
& \quad+\delta \alpha_{p}\left(p_{h}, p_{h}\right)+\alpha_{p}\left(p_{h}, \gamma^{-1} \operatorname{div} \boldsymbol{v}_{h}\right) .
\end{aligned}
$$

Next, we apply Young's inequality, use (4.13), and the coercivity of $a_{h}(\cdot, \cdot)$, to get

$$
\begin{aligned}
A_{h}\left(\left(\boldsymbol{u}_{h}, \boldsymbol{v}_{h}, p_{h}\right),\left(\boldsymbol{w}_{h}, \boldsymbol{z}_{h}, q_{h}\right)\right) \\
=\delta a_{h}\left(\boldsymbol{u}_{h}, \boldsymbol{u}_{h}\right)+\delta \lambda\left(\operatorname{div} \boldsymbol{u}_{h}, \operatorname{div} \boldsymbol{u}_{h}\right)+\delta R_{p}^{-1}\left(\boldsymbol{v}_{h}, \boldsymbol{v}_{h}\right)-\left(R_{p}^{-1 / 2} \boldsymbol{v}_{h}, \boldsymbol{v}_{h, 0}\right) \\
\quad+\left(p_{h}, R_{p} p_{h}\right)+\gamma^{-1}\left(\operatorname{div} \boldsymbol{u}_{h}, \operatorname{div} \boldsymbol{v}_{h}\right)+\gamma^{-1}\left(\operatorname{div} \boldsymbol{v}_{h}, \operatorname{div} \boldsymbol{v}_{h}\right) \\
\quad+\delta \alpha_{p}\left(p_{h}, p_{h}\right)+\alpha_{p}\left(p_{h}, \gamma^{-1} \operatorname{div} \boldsymbol{v}_{h}\right)
\end{aligned}
$$




$$
\begin{aligned}
& \geq \delta a_{h}\left(\boldsymbol{u}_{h}, \boldsymbol{u}_{h}\right)+\delta \lambda\left(\operatorname{div} \boldsymbol{u}_{h}, \operatorname{div} \boldsymbol{u}_{h}\right)+\delta R_{p}^{-1}\left(\boldsymbol{v}_{h}, \boldsymbol{v}_{h}\right)-\frac{1}{2} \epsilon_{1}^{-1} R_{p}^{-1}\left(\boldsymbol{v}_{h}, \boldsymbol{v}_{h}\right) \\
& \quad-\frac{1}{2} \epsilon_{1}\left(\boldsymbol{v}_{h, 0}, \boldsymbol{v}_{h, 0}\right)+R_{p}\left(p_{h}, p_{h}\right)-\frac{1}{2} \epsilon_{2}^{-1} \gamma^{-1}\left(\operatorname{div} \boldsymbol{u}_{h}, \operatorname{div} \boldsymbol{u}_{h}\right) \\
& \quad-\frac{1}{2} \epsilon_{2} \gamma^{-1}\left(\operatorname{div} \boldsymbol{v}_{h}, \operatorname{div} \boldsymbol{v}_{h}\right)+\gamma^{-1}\left(\operatorname{div} \boldsymbol{v}_{h}, \operatorname{div} \boldsymbol{v}_{h}\right)+\delta \alpha_{p}\left(p_{h}, p_{h}\right) \\
& \quad-\frac{1}{2} \epsilon_{3} \gamma^{-1}\left(\operatorname{div} \boldsymbol{v}_{h}, \operatorname{div} \boldsymbol{v}_{h}\right)-\frac{1}{2} \epsilon_{3}^{-1} \alpha_{p}^{2} \gamma^{-1}\left(p_{h}, p_{h}\right) \\
& \geq_{\text {by(4.8),(4.13) }} \delta \alpha_{a}\left\|\boldsymbol{u}_{h}\right\|_{D G}^{2}+\delta \lambda\left(\operatorname{div} \boldsymbol{u}_{h}, \operatorname{div} \boldsymbol{u}_{h}\right)-\frac{1}{2} \epsilon_{2}^{-1} \gamma^{-1}\left(\operatorname{div} \boldsymbol{u}_{h}, \operatorname{div} \boldsymbol{u}_{h}\right) \\
& \quad+\left(\delta-\frac{1}{2} \epsilon_{1}^{-1}\right) R_{p}^{-1}\left(\boldsymbol{v}_{h}, \boldsymbol{v}_{h}\right)+\left(1-\frac{1}{2} \epsilon_{2}-\frac{1}{2} \epsilon_{3}\right) \gamma^{-1}\left(\operatorname{div} \boldsymbol{v}_{h}, \operatorname{div} \boldsymbol{v}_{h}\right) \\
& \quad+\left(1-\frac{1}{2} \epsilon_{1} \beta_{d d}^{-2}\right) R_{p}\left(p_{h}, p_{h}\right)+\left(\delta-\frac{1}{2} \epsilon_{3}^{-1} \alpha_{p} \gamma^{-1}\right) \alpha_{p}\left(p_{h}, p_{h}\right) .
\end{aligned}
$$

Now, letting $\epsilon_{1}=\beta_{d d}^{2}, \epsilon_{2}=\epsilon_{3}=\frac{1}{2}$ and noting that $\rho^{-1} \leq \gamma, \lambda \geq \rho \geq \gamma^{-1}>0$, we obtain

$$
\begin{aligned}
& A_{h}\left(\left(\boldsymbol{u}_{h}, \boldsymbol{v}_{h}, p_{h}\right),\left(\boldsymbol{w}_{h}, \boldsymbol{z}_{h}, q_{h}\right)\right) \\
& \geq \delta \alpha_{a}\left\|\boldsymbol{u}_{h}\right\|_{D G}^{2}+(\delta-1) \lambda\left(\operatorname{div} \boldsymbol{u}_{h}, \operatorname{div} \boldsymbol{u}_{h}\right)+\left(\delta-\frac{1}{2} \beta_{d}^{-2}\right) R_{p}^{-1}\left(\boldsymbol{v}_{h}, \boldsymbol{v}_{h}\right) \\
& \quad+\frac{1}{2} \gamma^{-1}\left(\operatorname{div} \boldsymbol{v}_{h}, \operatorname{div} \boldsymbol{v}_{h}\right)+\frac{1}{2} R_{p}\left(p_{h}, p_{h}\right)+\left(\delta-\alpha_{p} \gamma^{-1}\right) \alpha_{p}\left(p_{h}, p_{h}\right) .
\end{aligned}
$$

Finally, we choose $\delta:=\max \left\{\frac{1}{2} \alpha_{a}^{-1}, \frac{1}{2} \beta_{d d}^{-2}+\frac{1}{2}, 1+\frac{1}{2}\right\}$, note that $\alpha_{p} \leq \gamma$, and conclude the coercivity of the bilinear form, i.e.,

$$
\begin{aligned}
& A_{h}\left(\left(\boldsymbol{u}_{h}, \boldsymbol{v}_{h}, p_{h}\right),\left(\boldsymbol{w}_{h}, \boldsymbol{z}_{h}, q_{h}\right)\right) \\
& \quad \geq \frac{1}{2}\left\|\boldsymbol{u}_{h}\right\|_{D G}^{2}+\frac{1}{2} \lambda\left(\operatorname{div} \boldsymbol{u}_{h}, \operatorname{div} \boldsymbol{u}_{h}\right)+\frac{1}{2} R_{p}^{-1}\left(\boldsymbol{v}_{h}, \boldsymbol{v}_{h}\right) \\
& \quad \quad+\frac{1}{2} \gamma^{-1}\left(\operatorname{div} \boldsymbol{v}_{h}, \operatorname{div} \boldsymbol{v}_{h}\right)+\frac{1}{2} R_{p}\left(p_{h}, p_{h}\right)+\frac{1}{2} \alpha_{p}\left(p_{h}, p_{h}\right) \\
& \quad \geq \frac{1}{2}\left(\left\|\boldsymbol{u}_{h}\right\|_{\boldsymbol{U}_{h}}^{2}+\left\|\boldsymbol{v}_{h}\right\|_{\boldsymbol{V}}^{2}+\left\|p_{h}\right\|_{P}^{2}\right) .
\end{aligned}
$$

This completes the proof.

From the above theorem, we get the following stability estimate.

COROLlaRY 4.5. Let $\left(\boldsymbol{u}_{h}, \boldsymbol{v}_{h}, p_{h}\right) \in \boldsymbol{U}_{h} \times \boldsymbol{V}_{h} \times P_{h}$ be the solution of (4.1), then we have the estimate

$$
\left\|\boldsymbol{u}_{h}\right\|_{\boldsymbol{U}_{h}}+\left\|\boldsymbol{v}_{h}\right\|_{\boldsymbol{V}}+\left\|p_{h}\right\|_{P} \leq C_{2}\left(\|\boldsymbol{f}\|_{\boldsymbol{U}_{h}^{*}}+\|g\|_{P^{*}}\right),
$$

where $\|\boldsymbol{f}\|_{\boldsymbol{U}_{h}^{*}}=\sup _{\boldsymbol{w}_{h} \in \boldsymbol{U}_{h}} \frac{\left(\boldsymbol{f}, \boldsymbol{w}_{h}\right)}{\left\|\boldsymbol{w}_{h}\right\|_{U_{h}}},\|g\|_{P^{*}}=\sup _{q_{h} \in P_{h}} \frac{\left(g, q_{h}\right)}{\left\|q_{h}\right\|_{P}}$, and $C_{2}$ is a constant independent of $\lambda, R_{p}^{-1}, \alpha_{p}$ and the mesh size $h$.

REMARK 4.6. Denote by $\mathcal{A}_{h}$ the operator induced by the bilinear form (4.9), namely

$$
\mathcal{A}_{h}:=\left[\begin{array}{ccc}
-\operatorname{div}_{h} \epsilon_{h}-\lambda \nabla_{h} \operatorname{div}_{h} & 0 & \nabla_{h} \\
0 & R_{p}^{-1} I_{h} & \nabla_{h} \\
-\operatorname{div}_{h} & -\operatorname{div}_{h} & -\alpha_{p} I_{h}
\end{array}\right],
$$

and define

$$
\mathcal{B}_{h}:=\left[\begin{array}{ccc}
\left(-\operatorname{div}_{h} \boldsymbol{\epsilon}_{h}-\lambda \nabla_{h} \operatorname{div}_{h}\right)^{-1} & 0 & 0 \\
0 & \left(R_{p}^{-1} I_{h}+\gamma^{-1} \nabla_{h} \operatorname{div}_{h}\right)^{-1} & 0 \\
0 & 0 & \left(\gamma I_{h}\right)^{-1}
\end{array}\right]
$$


Then due to the theory presented in [29], Theorems 4.3 and 4.4 imply that the norm-equivalent (canonical) block-diagonal preconditioner $\mathcal{B}_{h}$ for $\mathcal{A}_{h}$ is parameter-robust, which means that the condition number $\mathcal{K}\left(\mathcal{B}_{h} \mathcal{A}_{h}\right)$ is uniformly bounded with respect to the parameters $\lambda, R_{p}^{-1}, \alpha_{p}$ in the ranges specified in (3.1) and with respect to the mesh size $h$. To apply the preconditioner $\mathcal{B}_{h}$, one has to solve an elasticity system discretized by an $H(\mathrm{div})$-conforming discontinuous Galerkin method and an elliptic $H$ (div) problem discretized by $R T$ elements. In the lowest order case, optimal solvers for these tasks have been proposed in [23, 25].

5. Error estimates. In this section, we derive error estimates that follow from the results presented in Section 4. Let $\Pi_{B}^{\text {div }}: H^{1}(\Omega)^{d} \mapsto \boldsymbol{U}_{h}$ be the canonical interpolation operator. We also denote the $L^{2}$-projection on $P_{h}$ by $Q_{h}$. The following Lemma summarizes some of the properties of $\Pi_{B}^{\text {div }}$ and $Q_{h}$ needed for our proof; see [23].

LEMMA 5.1. For all $\boldsymbol{w} \in H^{1}(K)^{d}$ we have

$$
\operatorname{div} \Pi_{B}^{\mathrm{div}}=Q_{h} \operatorname{div}, \quad\left|\Pi_{B}^{\mathrm{div}} \boldsymbol{w}\right|_{1, K} \lesssim|\boldsymbol{w}|_{1, K}, \quad\left\|\boldsymbol{w}-\Pi_{B}^{\mathrm{div}} \boldsymbol{w}\right\|_{0, \partial K}^{2} \lesssim h_{K}|\boldsymbol{w}|_{1, K}^{2} .
$$

THEOREM 5.2. Let $(\boldsymbol{u}, \boldsymbol{v}, p)$ be the solution of (2.4) and $\left(\boldsymbol{u}_{h}, \boldsymbol{v}_{h}, p_{h}\right)$ be the solution of (4.1). Then the error estimates

$$
\left\|\boldsymbol{u}-\boldsymbol{u}_{h}\right\|_{\boldsymbol{U}_{h}}+\left\|\boldsymbol{v}-\boldsymbol{v}_{h}\right\|_{\boldsymbol{V}} \leq C_{e, u} \inf _{\boldsymbol{w}_{h} \in \boldsymbol{U}_{h}, \boldsymbol{z}_{h} \in \boldsymbol{V}_{h}}\left(\left\|\boldsymbol{u}-\boldsymbol{w}_{h}\right\|_{\boldsymbol{U}_{h}}+\left\|\boldsymbol{v}-\boldsymbol{z}_{h}\right\|_{\boldsymbol{V}}\right)
$$

and

$$
\left\|p-p_{h}\right\|_{P} \leq C_{e, p} \inf _{\boldsymbol{w}_{h} \in \boldsymbol{U}_{h}, \boldsymbol{z}_{h} \in \boldsymbol{V}_{h}, q_{h} \in P_{h}}\left(\left\|\boldsymbol{u}-\boldsymbol{w}_{h}\right\|_{\boldsymbol{U}_{h}}+\left\|\boldsymbol{v}-\boldsymbol{z}_{h}\right\|_{\boldsymbol{V}}+\left\|p-q_{h}\right\|_{P}\right)
$$

hold, where $C_{e, u}, C_{e, p}$ are constants independent of $\lambda, R_{p}^{-1}, \alpha_{p}$ and the mesh size $h$.

Proof. Subtracting (4.1) from (2.4) and noting the consistency of $a_{h}(\cdot, \cdot)$, we have that for any $\left(\boldsymbol{w}_{h}, \boldsymbol{z}_{h}, q_{h}\right) \in \boldsymbol{U}_{h} \times \boldsymbol{V}_{h} \times P_{h}$

$$
\begin{array}{r}
a_{h}\left(\boldsymbol{u}-\boldsymbol{u}_{h}, \boldsymbol{w}_{h}\right)+\lambda\left(\operatorname{div}\left(\boldsymbol{u}-\boldsymbol{u}_{h}\right), \operatorname{div} \boldsymbol{w}_{h}\right)-\left(\left(p-p_{h}\right), \operatorname{div} \boldsymbol{w}_{h}\right)=0, \\
R_{p}^{-1}\left(\boldsymbol{v}-\boldsymbol{v}_{h}, \boldsymbol{z}_{h}\right)-\left(p-p_{h}, \operatorname{div} \boldsymbol{z}_{h}\right)=0, \\
-\left(\operatorname{div}\left(\boldsymbol{u}-\boldsymbol{u}_{h}\right), q_{h}\right)-\left(\operatorname{div}\left(\boldsymbol{v}-\boldsymbol{v}_{h}\right), q_{h}\right)-\alpha_{p}\left(p-p_{h}, q_{h}\right)=0 .
\end{array}
$$

Let $\boldsymbol{u}_{I}=\Pi_{B}^{\text {div }} \boldsymbol{u} \in \boldsymbol{U}_{h}, p_{I}=Q_{h} p \in P_{h}$. Now for arbitrary $\boldsymbol{v}_{I} \in \boldsymbol{V}_{h}$, from (5.1), noting that $\operatorname{div} \Pi_{B}^{\text {div }}=Q_{h} \operatorname{div}$ and $\operatorname{div} \boldsymbol{U}_{h}=\operatorname{div} \boldsymbol{V}_{h}=P_{h}$, we conclude

$$
\begin{aligned}
a_{h}\left(\boldsymbol{u}_{I}-\boldsymbol{u}_{h}, \boldsymbol{w}_{h}\right)+\lambda\left(\operatorname{div}\left(\boldsymbol{u}_{I}-\boldsymbol{u}_{h}\right), \operatorname{div} \boldsymbol{w}_{h}\right)-\left(p_{I}-p_{h}, \operatorname{div} \boldsymbol{w}_{h}\right) & =a_{h}\left(\boldsymbol{u}_{I}-\boldsymbol{u}, \boldsymbol{w}_{h}\right), \\
R_{p}^{-1}\left(\boldsymbol{v}_{I}-\boldsymbol{v}_{h}, \boldsymbol{z}_{h}\right)-\left(p_{I}-p_{h}, \operatorname{div} \boldsymbol{z}_{h}\right) & =R_{p}^{-1}\left(\boldsymbol{v}_{I}-\boldsymbol{v}, \boldsymbol{z}_{h}\right), \\
-\left(\operatorname{div}\left(\boldsymbol{u}_{I}-\boldsymbol{u}_{h}\right), q_{h}\right)-\left(\operatorname{div}\left(\boldsymbol{v}_{I}-\boldsymbol{v}_{h}\right), q_{h}\right)-\alpha_{p}\left(p_{I}-p_{h}, q_{h}\right) & =-\left(\operatorname{div}\left(\boldsymbol{v}_{I}-\boldsymbol{v}\right), q_{h}\right) .
\end{aligned}
$$

Next, since $\left(\boldsymbol{u}_{I}-\boldsymbol{u}_{h}\right) \in \boldsymbol{U}_{h},\left(\boldsymbol{v}_{I}-\boldsymbol{v}_{h}\right) \in \boldsymbol{V}_{h},\left(p_{I}-p_{h}\right) \in P_{h}$, by the stability result (4.10) for the discrete problem (4.1), we obtain

$$
\begin{aligned}
& \left\|\boldsymbol{u}_{I}-\boldsymbol{u}_{h}\right\|_{\boldsymbol{U}_{h}}+\left\|\boldsymbol{v}_{I}-\boldsymbol{v}_{h}\right\|_{\boldsymbol{V}} \\
& \quad \leq C_{e}\left(\sup _{\boldsymbol{w}_{h} \in \boldsymbol{U}_{h}} \frac{a_{h}\left(\boldsymbol{u}_{I}-\boldsymbol{u}, \boldsymbol{w}_{h}\right)}{\left\|\boldsymbol{w}_{h}\right\|_{\boldsymbol{U}_{h}}}+\sup _{\boldsymbol{z}_{h} \in \boldsymbol{V}_{h}} \frac{R_{p}^{-1}\left(\boldsymbol{v}_{I}-\boldsymbol{v}, \boldsymbol{z}_{h}\right)}{\left\|\boldsymbol{z}_{h}\right\|_{\boldsymbol{V}}}+\sup _{q_{h} \in P_{h}} \frac{\left(\operatorname{div}\left(\boldsymbol{v}-\boldsymbol{v}_{I}\right), q_{h}\right)}{\left\|q_{h}\right\|_{P}}\right), \\
& \left\|p_{I}-p_{h}\right\|_{P} \\
& \quad \leq C_{e}\left(\sup _{\boldsymbol{w}_{h} \in \boldsymbol{U}_{h}} \frac{a_{h}\left(\boldsymbol{u}_{I}-\boldsymbol{u}, \boldsymbol{w}_{h}\right)}{\left\|\boldsymbol{w}_{h}\right\|_{\boldsymbol{U}_{h}}}+\sup _{\boldsymbol{z}_{h} \in \boldsymbol{V}_{h}} \frac{R_{p}^{-1}\left(\boldsymbol{v}_{I}-\boldsymbol{v}, \boldsymbol{z}_{h}\right)}{\left\|\boldsymbol{z}_{h}\right\|_{\boldsymbol{V}}}+\sup _{q_{h} \in P_{h}} \frac{\left(\operatorname{div}\left(\boldsymbol{v}-\boldsymbol{v}_{I}\right), q_{h}\right)}{\left\|q_{h}\right\|_{P}}\right) .
\end{aligned}
$$


Using the boundedness of $a_{h}(\cdot, \cdot)$, the second inequality in Lemma 5.1, the triangle inequality, the fact that $\boldsymbol{v}_{I}$ is arbitrary, and $\left(\operatorname{div}\left(\boldsymbol{v}-\boldsymbol{v}_{I}\right), q_{h}\right) \leq\left\|\boldsymbol{v}-\boldsymbol{v}_{I}\right\|_{\boldsymbol{V}}\left\|q_{h}\right\|_{P}$, we arrive at

$$
\left\|\boldsymbol{u}-\boldsymbol{u}_{h}\right\|_{\boldsymbol{U}_{h}}+\left\|\boldsymbol{v}-\boldsymbol{v}_{h}\right\|_{\boldsymbol{V}} \leq C_{e, u} \inf _{\boldsymbol{w}_{h} \in \boldsymbol{U}_{h}, \boldsymbol{z}_{h} \in \boldsymbol{V}_{h}}\left(\left\|\boldsymbol{u}-\boldsymbol{w}_{h}\right\|_{\boldsymbol{U}_{h}}+\left\|\boldsymbol{v}-\boldsymbol{z}_{h}\right\|_{\boldsymbol{V}}\right),
$$

and

$\left\|p-p_{h}\right\|_{P} \leq C_{e, p} \inf _{\boldsymbol{w}_{h} \in \boldsymbol{U}_{h}, \boldsymbol{z}_{h} \in \boldsymbol{V}_{h}, q_{h} \in P_{h}}\left(\left\|\boldsymbol{u}-\boldsymbol{w}_{h}\right\|_{\boldsymbol{U}_{h}}+\left\|\boldsymbol{v}-\boldsymbol{z}_{h}\right\|_{\boldsymbol{V}}+\left\|p-q_{h}\right\|_{P}\right)$.

REMARK 5.3. From the above theorem, we can see that the discretizations are lockingfree.

6. Conclusions. This paper presents the stability analysis of a classical three-field formulation of Biot's consolidation model where the unknown variables are the displacements, the fluid flux (Darcy velocity), and the pore pressure. Specific parameter-dependent norms provide the key to establish the parameter-robust stability of the continuous problem. This allows for the construction of a parameter-robust block diagonal preconditioner in the framework of operator preconditioning. Discretizations that provide strong mass conservation are designed. Further, both discrete parameter-robust stability and locking-free error estimates are proved.

\section{REFERENCES}

[1] J. Adler, F. Gaspar, X. Hu, C. Rodrigo, And L. Zikatanov, Robust block preconditioners for Biot's model, Preprint on arXiv, 2017. https://arxiv.org/abs/1705.08842.

[2] D. N. ARNOLD, An interior penalty finite element method with discontinuous elements, SIAM J. Numer. Anal., 19 (1982), pp. 742-760.

[3] D. N. ARnold, F. Brezzi, B. Cockburn, And L. D. Marini, Unified analysis of discontinuous Galerkin methods for elliptic problems, SIAM J. Numer. Anal., 39 (2001/02), pp. 1749-1779.

[4] O. Axelsson, R. Blaheta, AND P. BycZAnski, Stable discretization of poroelasticity problems and efficient preconditioners for arising saddle point type matrices, Comput. Vis. Sci., 15 (2012), pp. 191-207.

[5] I. BABUŠKA, Error-bounds for finite element method, Numer. Math., 16 (1970/1971), pp. 322-333.

[6] T. BÆRLAND, J. J. LEE, K.-A. MARDAL, AND R. WINTHER, Weakly imposed symmetry and robust preconditioners for Biot's consolidation model, Comput. Methods Appl. Math., 17 (2017), pp. 377-396.

[7] M. А. ВІот, General theory of three-dimensional consolidation, J. Appl. Phys., 12 (1941), pp. 155-164.

[8] — Theory of elasticity and consolidation for a porous anisotropic solid, J. Appl. Phys., 26 (1955), pp. 182-185.

[9] D. BOFFI, F. BREZZI, AND M. ForTIN, Mixed Finite Element Methods and Applications, Springer, Heidelberg, 2013.

[10] S. C. BRENnER, Korn's inequalities for piecewise $H^{1}$ vector fields, Math. Comp., 73 (2004), pp. 1067-1087.

[11] F. BREZZI, On the existence, uniqueness and approximation of saddle-point problems arising from Lagrangian multipliers, Rev. Française Automat. Informat. Recherche Opérationnelle Sér. Rouge, 8 (1974), pp. 129151.

[12] F. BrezZi, G. Manzini, D. Marini, P. Pietra, And A. Russo, Discontinuous Galerkin approximations for elliptic problems, Numer. Methods Partial Differential Equations, 16 (2000), pp. 365-378.

[13] B. Cockburn, G. KAnSchat, AND D. SchötZAU, A note on discontinuous Galerkin divergence-free solutions of the Navier-Stokes equations, J. Sci. Comput., 31 (2007), pp. 61-73.

[14] O. Coussy, Poromechanics, Wiley, Chichester, 2004.

[15] E. Detrounay and A.H.-D. Cheng, Fundamentals of poroelasticity, in Comprehensive Rock Engineering: Principles, Practice and Projects, Vol. II, Analysis and Design Methods, C. Fairhurst, ed., Pergamon Press, Oxford, 1993, pp. 113-171.

[16] A. ERn And J.-L. Guermond, Theory and Practice of Finite Elements, Springer, New York, 2004.

[17] M. ForTIN AND M. SOULIE, A nonconforming piecewise quadratic finite element on triangles, Internat. J. Numer. Methods Engrg., 19 (1983), pp. 505-520.

[18] F. J. Gaspar, F. J. Lisbona, AND P. N. VABISHChEVICH, A finite difference analysis of Biot's consolidation model, Appl. Numer. Math., 44 (2003), pp. 487-506.

[19] - Staggered grid discretizations for the quasi-static Biot's consolidation problem, Appl. Numer. Math., 56 (2006), pp. 888-898. 
[20] P. Hansbo And M. G. LaRson, Discontinuous Galerkin and the Crouzeix-Raviart element: application to elasticity, M2AN Math. Model. Numer. Anal., 37 (2003), pp. 63-72.

[21] Q. Hong, J. HU, S. SHU, AND J. XU, A discontinuous Galerkin method for the fourth-order curl problem, J. Comput. Math., 30 (2012), pp. 565-578.

[22] Q. Hong AND J. KRAUS, Uniformly stable discontinuous Galerkin discretization and robust iterative solution methods for the Brinkman problem, SIAM J. Numer. Anal., 54 (2016), pp. 2750-2774.

[23] Q. Hong, J. KRAus, J. XU, AND L. ZiKatanov, A robust multigrid method for discontinuous Galerkin discretizations of Stokes and linear elasticity equations, Numer. Math., 132 (2016), pp. 23-49.

[24] X. Hu, C. Rodrigo, F. J. Gaspar, And L. T. ZiKatanov, A nonconforming finite element method for the Biot's consolidation model in poroelasticity, J. Comput. Appl. Math., 310 (2017), pp. 143-154.

[25] J. Kraus, R. LaZarov, M. Lymbery, S. Margenov, And L. Zikatanov, Preconditioning heterogeneous $H$ (div) problems by additive Schur complement approximation and applications, SIAM J. Sci. Comput., 38 (2016), pp. A875-A898.

[26] J. J. LEE, Robust error analysis of coupled mixed methods for Biot's consolidation model, J. Sci. Comput., 69 (2016), pp. 610-632.

[27] J. J. LEE, K.-A. MARDAL, AND R. WinTHER, Parameter-robust discretization and preconditioning of Biot's consolidation model, SIAM J. Sci. Comput., 39 (2017), pp. A1-A24.

[28] K. LiPniKov, Numerical Methods for the Biot Model in Poroelasticity, PhD. Thesis, Dept. of Mathematics, University of Houston, Houston, 2002.

[29] K.-A. Mardal And R. Winther, Preconditioning discretizations of systems of partial differential equations, Numer. Linear Algebra Appl., 18 (2011), pp. 1-40.

[30] J. M. Nordbotten, Stable cell-centered finite volume discretization for Biot equations, SIAM J. Numer. Anal., 54 (2016), pp. 942-968.

[31] R. OYARZÚA AND R. RUIZ-BAIER, Locking-free finite element methods for poroelasticity, SIAM J. Numer. Anal., 54 (2016), pp. 2951-2973.

[32] P. J. Phillips AND M. F. WheEler, A coupling of mixed and continuous Galerkin finite element methods for poroelasticity. I. The continuous in time case, Comput. Geosci., 11 (2007), pp. 131-144.

[33] - A coupling of mixed and discontinuous Galerkin finite-element methods for poroelasticity, Comput. Geosci., 12 (2008), pp. 417-435.

[34] D. Schötzau, C. Schwab, And A. Toselli, Mixed hp-DGFEM for incompressible flows, SIAM J. Numer. Anal., 40 (2002), pp. 2171-2194 (2003).

[35] J. Smith And J. Humphrey, Interstitial transport and transvascular fluid exchange during infusion into brain and tumor tissue, Microvasc. Res., 73 (2007), pp. 58-73.

[36] K. H. Støverud, M. Alnæs, H. Langtangen, V. Haughton, and K.-A. Mardal, Poro-elastic modeling of Syringomyelia - a systematic study of the effects of pia mater, central canal, median fissure white and gray matter on pressure wave propagation and fluid movement within the cervical spinal cord, Comput. Methods Biomech. Biomed. Engin., 19 (2016), pp. 686-698.

[37] H. WANG, Theory of Linear Poroelasticity with Applications to Geomechanics and Hydrogeology, Princeton University Press, Princeton, 2000. 